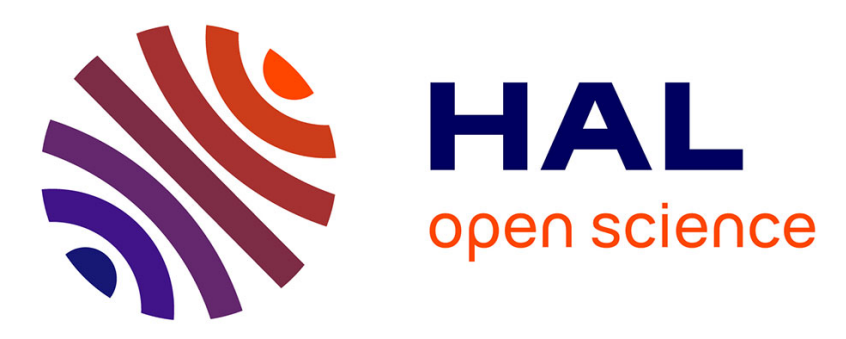

\title{
Screening of marine microalgae: Investigation of new exopolysaccharide producers
}

Clément Gaignard, Céline Laroche, Guillaume Pierre, Pascal Dubessay, Cédric Delattre, Christine Gardarin, Priscilla Gourvil, Ian Probert, Aurore Dubuffet, Philippe Michaud

\section{To cite this version:}

Clément Gaignard, Céline Laroche, Guillaume Pierre, Pascal Dubessay, Cédric Delattre, et al.. Screening of marine microalgae: Investigation of new exopolysaccharide producers. Algal Research - Biomass, Biofuels and Bioproducts, 2019, 44, pp.101711. 10.1016/j.algal.2019.101711 . hal-02364692

\section{HAL Id: hal-02364692 https://hal.science/hal-02364692}

Submitted on 18 Nov 2019

HAL is a multi-disciplinary open access archive for the deposit and dissemination of scientific research documents, whether they are published or not. The documents may come from teaching and research institutions in France or abroad, or from public or private research centers.
L'archive ouverte pluridisciplinaire HAL, est destinée au dépôt et à la diffusion de documents scientifiques de niveau recherche, publiés ou non, émanant des établissements d'enseignement et de recherche français ou étrangers, des laboratoires publics ou privés. 

C. Gaignard ${ }^{1}$,
C. Laroche ${ }^{1}$,
G. Pierre' ${ }^{1}$ P. Dubessay ${ }^{1}$,
C. Delattre ${ }^{1}$, C.

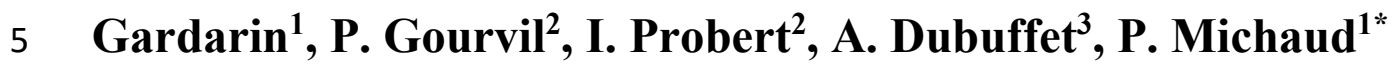

6

7 '1 Université Clermont Auvergne, CNRS, SIGMA Clermont, Institut Pascal, F-63000 CLERMONT-FERRAND,

8 France.

$9 \quad{ }^{2}$ Station Biologique de Roscoff (SBR), FR2424, Sorbonne Université, CNRS, Place Georges Teissier, 29680

10 Roscoff, France.

$11{ }^{3}$ Université Clermont Auvergne, CNRS, Laboratoire Microorganismes : Génome et Environnement, F-63000

12 CLERMONT-FERRAND, France.

15 Corresponding author: *Philippe MICHAUD: philippe.michaud@uca.fr

Tel.: +33(0)473407425 
Abstract:

Biopolymers, such as exopolysaccharides are widely exploited by industry as hydrocolloids (gelling, thickening agents) and biological agents (anti-inflammatory, anti-parasitic, antioxidant, etc.). In this study, 166 marine microalgae and cyanobacteria species have been screened in order to identify strains producing original exopolysaccharides. This screening allowed the highlighting of 45 positive strains. In a second time, the monosaccharide compositions from 20 EPS of them were determined by GC/MS and HPAEC-PAD. The results led to a discovery of 8 new genera of microalgae producing EPS, including polymers with a very original composition like richness in GlcA. Finally, a phylogenic tree has been contructed in order to assess the link between the phylogeny of microalgae and the global composition of their exopolymers, based on data obtained in this study and from the literature.

Keywords: Screening, Microalgae, Polysaccharides, Exopolysaccharides, Phylogeny 


\section{Introduction}

Microalgae and cyanobacteria are single-cell photoautotrophic microorganisms which can produce biomass with a great efficiency using solar energy, water and $\mathrm{CO}_{2}$. These organisms represent a huge diversified group including theoretically between 200000 and 800 000 different species, 35000 of them being really identified in marine and fresh water environments but also acidic lakes, hypersaline waters, or desert areas [1]. These photosynthetic microorganisms can be divided into blue-green algae or cyanobacteria and major eukaryotic groups including green microalgae (Chlorophyta and Charophyta), red microalgae (Rhodophyta) and brown-golden microalgae (Ochrophyta, Haptophyta and Miozoa). This large taxonomic diversity makes microalgae very attractive to produce valuable and diverse biomolecules such as pigments, proteins including some enzymes, PolyUnsaturated Fatty Acids (PUFAs), lipids, and exopolysaccharides (EPS). These compounds present some interests for many diverse industrial areas, like pharmaceutical, nutraceutical, cosmetic and food/feed production [2-3]. Moreover, the development and commercialization of these metabolites is still early and only niche markets are currently available for microalgae products. Their low usage is very surprising but may be easily explained by the costs involved in microalgae production, limiting their applications to the field of high value products. These costs are linked to the photoproduction, the harvest of microalgae in diluted media, the recycling of culture media and the difficulty to refine this biomass [4-5].

Among the high value compounds from microalgae, EPS are very promising. Even if polysaccharides from animals, fungi and higher plants (including macroalgae) have been studied for many years, interest of the scientific community for EPS produced by microalgae and cyanobacteria is much more recent as described by Rossi and De Philippis [6] and Delattre et al. [7]. Based on their function, polysaccharides from photosynthetic microorganisms can be divided into three families: i) structural polysaccharides from cell walls, ii) storage 
polysaccharides which are intracellular and iii) matricial polysaccharides released into the environment. Some of them are not completely released into the medium and remain linked to the cell. So, matricial polysaccharides were split into two categories by authors: i) those which are retained to the cell and called cell-bound polysaccharides or capsular polysaccharides and ii) those completely released into the environment called, extracellular polysaccharides, extracellular polymeric substance or exopolysaccharides (EPS). Today, there is always a thin line between these categories and the use of "EPS" is often clumsily used to qualify cell bound polysaccharides. The first publication focusing on EPS from microalgae was published in 1964 by Tischer and Moore and revealed the composition of a polymer produced by Palmella mucosa [8]. Since the number of species of microalgae/cyanobacteria producing EPS described in literature has increased at a moderate level. Even if the effects of environment (culture conditions) on the EPS production by microalgae and cyanobacteria appear as species dependent, nutrient limitation and notably nitrogen starvation is the most described environmental constraint leading to EPS synthesis [7]. The main families characterized are Desmidiaceae, Chlamydomonadaceae, Chlorellaceae, Porphyridiaceae and Glaucosphaeraceae in microalgae, and Nostocaceae, Oscillatoriaceae and Microcoleaceae in cyanobacteria. Note also that some microalgae belonging to the Chromista kingdom and more especially those from the Bacilariophyceae class have also been described for the production of EPS. This rise is not really correlated with the large taxonomic diversity of microalgae despite the detection of in vivo and in vitro biological activities associated to the presence of these biopolymers such as immunomodulatory [9], anti-oxidative [10], anti-inflammatory [11], anticoagulant [12], antibacterial [13], anticancer [14], or anti-parasitic [15] properties. Some interesting rheological properties revealed also the potential of these EPS as gelling and thickening agents [7]. Based on these observations but also on the large diversity of unstudied microalgae, these microorganisms are very attractive regarding the production of new EPS. 

microalgae/cyanobacteria producing original EPS and to screen them for this phenotype. It has been done as part of the French Polysalgue project funded by the French National Research Agency (ANR) and aims to identify new strains of microalgae and cyanobacteria for the production of high value EPS. Previous screening studies had been done by De Philippis et al. $[\mathbf{1 6}, \mathbf{1 7 ]}$ but it was restricted to cyanobacteria, in 15 and 25 strains of the Cyanothece and Nostoc genera, respectively. In this work 166 original strains including 150 microalgae and 16 cyanobacteria from diverse phylogenetic groups collected and distributed by the Roscoff Culture Collection (RCC), France (http://roscoff-culture-collection.org/) were screened for the production of EPS. Strains were selected based on their phylogenic diversity, their promising phenotype and their belonging to species not previously described as EPS producers. Positive strains were able to growth in the under chosing conditions of culture and to produce a minimum of 0.05 g. $\mathrm{L}^{-1}$ of extracellular carbohydrates after 30 days of culture. The results obtained were discussed and compared to the literature in order to highlight a possible relationship between phylogenetic microalgae groups and EPS production/composition.

\section{Material and methods}

\subsection{Screening procedure}

The screening was performed on 166 marine microalgae and cyanobacteria species from the RCC (Table 1 and Supplementary data 1 Figure S1). The f/2 medium was used for microalgae cultivation [18]. It was composed of $\mathrm{NaNO}_{3} 0.075 \mathrm{~g}, \mathrm{NaH}_{2} \mathrm{PO}_{4} .2 \mathrm{H}_{2} \mathrm{O} 0.0055 \mathrm{~g}$, $\mathrm{Na}_{2}$ EDTA $0.00416 \mathrm{~g}, \mathrm{FeCl}_{3} .6 \mathrm{H}_{2} \mathrm{O} 0.00315 \mathrm{~g}, \mathrm{CuSO}_{4} .5 \mathrm{H}_{2} \mathrm{O} 0.00001 \mathrm{~g}, \mathrm{ZnSO}_{4} .7 \mathrm{H}_{2} \mathrm{O} 0.000022$ g, $\mathrm{CoCl}_{2} .6 \mathrm{H}_{2} \mathrm{O} \quad 0.00001$ g, $\mathrm{MnCl}_{2} .4 \mathrm{H}_{2} \mathrm{O} \quad 0.00018$ g, $\mathrm{Na}_{2} \mathrm{MoO}_{4} .2 \mathrm{H}_{2} \mathrm{O} \quad 0.000006$ g, cyanocobalamin (B12) $0.0000005 \mathrm{~g}$, thiamin (B1) $0.0001 \mathrm{~g}$ and biotin $0.0000005 \mathrm{~g}$ dissolved in $1 \mathrm{~L}$ of artificial sea water (ASW). ASW was composed of $\mathrm{NaCl} 28.13 \mathrm{~g}, \mathrm{KCl} 0.77 \mathrm{~g}$, $\mathrm{CaCl}_{2} .2 \mathrm{H}_{2} \mathrm{O} 1.60 \mathrm{~g}, \mathrm{MgCl}_{2} .6 \mathrm{H}_{2} \mathrm{O} 4.80 \mathrm{~g}, \mathrm{NaHCO}_{3} 0.11 \mathrm{~g}, \mathrm{MgSO}_{4} .7 \mathrm{H}_{2} \mathrm{O} 3.50 \mathrm{~g}$ dissolved in 1 
L of milliQ water. The $\mathrm{pH}$ was adjusted to $8-8.2$ and the medium was sterilized by filtration $(0.22 \mu \mathrm{m})$. The medium used for cyanobacteria was the PCR-S11 described by the RCC (http://roscoff-culture-collection.org/medium-id/pcr-s11-red-sea) and adapted from [19]. It was composed of one liter of Red Sea salt solution at 33.3 g.L $\mathrm{L}^{-1}$ supplemented by $1 \mathrm{~mL}$ of HEPES$\mathrm{NaOH} 1 \mathrm{M}(\mathrm{pH} 7.5)$ buffer, $1 \mathrm{~mL}$ of $\mathrm{Na}_{2}-\mathrm{EDTA} / \mathrm{FeCl}_{3}$ solution, $1 \mathrm{~mL}$ of $\mathrm{Na}^{-\mathrm{PO}_{4}} 50 \mathrm{mM}(\mathrm{pH}$ 7.5) buffer, $1 \mathrm{~mL}$ of $\left(\mathrm{NH}_{4}\right)_{2} \mathrm{SO}_{4}$ solution $(400 \mathrm{mM}), 100 \mu \mathrm{L}$ of trace metals « Gaffron+Se » and $100 \mu \mathrm{L}$ of vitamin B12 (10 mg.mL $\left.\mathrm{m}^{-1}\right)$. The red Sea salt solution was sterilised at $121^{\circ} \mathrm{C}$ during 20 min whereas the other solutions were filet-sterilized $(0.22 \mu \mathrm{m})$. The HEPES-NaOH $1 \mathrm{M}(\mathrm{pH}$ 7.5) buffer was composed of $119.5 \mathrm{~g}$ of HEPES dissolved in $250 \mathrm{~mL}$ of milliQ water. The $\mathrm{pH}$ of the solution was adjusted to 7.5 before diluting with milliQ water to a final volume of 500 $\mathrm{mL}$. The $\mathrm{Na}_{2}$-EDTA/ $\mathrm{FeCl}_{3}$ complex solution was prepared dissolving $\mathrm{FeCl}_{3} \cdot 6 \mathrm{H}_{2} \mathrm{O}(1.08 \mathrm{~g})$ and $\mathrm{Na}_{2}$-EDTA $(1.488 \mathrm{~g})$ in $40 \mathrm{ml}$ of $\mathrm{HCl}(0.1 \mathrm{M})$ and $40 \mathrm{ml}$ of $\mathrm{NaOH}(0.1 \mathrm{M})$, respectively, before mixing and diluting with milliQ water to a final volume of $2000 \mathrm{~mL}$. The « Gaffron+Se » trace metal solution used contained for one litre of milliQ water $\mathrm{H}_{3} \mathrm{BO}_{3}(0.186 \mathrm{~g}), \mathrm{MnSO}_{4} \cdot \mathrm{H}_{2} \mathrm{O}(0.101$ g), $\left(\mathrm{NH}_{4}\right)_{6} \mathrm{MO}_{7} \mathrm{O}_{24 .} 4 \mathrm{H}_{2} \mathrm{O}(0.00198 \mathrm{~g}), \mathrm{KBr}(0.00516), \mathrm{KI}(0.00714 \mathrm{~g}), \mathrm{ZnSO}_{4} .7 \mathrm{H}_{2} \mathrm{O}(0.00498$ g), $\left(\mathrm{Cd}\left(\mathrm{NO}_{3}\right)_{2} .4 \mathrm{H}_{2} \mathrm{O}(0.01725 \mathrm{~g}), \mathrm{Co}\left(\mathrm{NO}_{3}\right)_{2} .6 \mathrm{H}_{2} \mathrm{O}(0.00925 \mathrm{~g}), \mathrm{CuSO}_{4} .5 \mathrm{H}_{2} \mathrm{O}(0.00876 \mathrm{~g})\right.$, $\left.\begin{array}{llllllll}\mathrm{NiCl}_{2} .6 \mathrm{H}_{2} \mathrm{O} & (0.0071 & \mathrm{g}\end{array}\right), \quad \mathrm{Cr}\left(\mathrm{NO}_{3}\right)_{3} .9 \mathrm{H}_{2} \mathrm{O} \quad(0.0024 \mathrm{~g}), \quad \mathrm{VOSO}_{4} .5 \mathrm{H}_{2} \mathrm{O} \quad(0,0015 \mathrm{~g})$, $\mathrm{KAl}\left(\mathrm{SO}_{4}\right) 2.12 \mathrm{H}_{2} \mathrm{O}(0.0284 \mathrm{~g})$ and $\mathrm{SeO}_{2}(0.0033 \mathrm{~g})$. Finally, the $\mathrm{Na}-\mathrm{PO}_{4} 50 \mathrm{mM}(\mathrm{pH} 7.5)$ buffer was composed of $6 \mathrm{~g}$ of $\mathrm{NaH}_{2} \mathrm{PO}_{4}$ dissolved in 1 litre of milliQ water mixed with $3.55 \mathrm{~g}$ of $\mathrm{Na}_{2} \mathrm{HPO}_{4}$ dissolved in $500 \mathrm{~mL}$ of milliQ water. The $\mathrm{pH}$ of the buffer was then adjusted to 7.5. Strains were cultivated photoautotrophically during 30 days in $50 \mathrm{~mL}$ flasks containing $20 \mathrm{~mL}$ of medium inoculated with $4 \mathrm{~mL}$ of microorganism culture. Cultures were performed at $20{ }^{\circ} \mathrm{C}$ under stirring (120 rpm). The values of irradiance were 150 and $300 \mu \mathrm{mol} \mathrm{m}{ }^{-2} \cdot \mathrm{s}^{-1}$ in parallel with a light:dark regime (16h: $8 \mathrm{~h})$. These irradiance values were chosen in order to be suitable for strains growing at low $\left(150 \mu \mathrm{mol} \mathrm{m} \mathrm{m}^{-2} \cdot \mathrm{s}^{-1}\right)$ and high $\left(300 \mu \mathrm{mol} \mathrm{m} \mathrm{m}^{-2} \cdot \mathrm{s}^{-1}\right)$ light intensities in 
accordance with literature $[3,7]$. This also allowed studying the impact of the light intensity on the production of EPS for further experiments. Samples of $1 \mathrm{~mL}$ were collected throughout the process to follow cell growth, nitrate consumption and extracellular sugars production.

\subsection{Growth, nitrate consumption and EPS production measurements}

Growth of microalgae/cyanobacteria was followed by spectrophotometry $\left(\mathrm{A}_{750}\right)$. This wavelength was chosen to avoid interferences with the pigments. Nitrate and ammonium concentrations were measured on supernatant of culture samples treated by centrifugation (10 $000 \mathrm{~g}$ for $10 \mathrm{~min}$ at $20^{\circ} \mathrm{C}$ ) according to the method of Caswe et al. [20] modified by A.P.H.A [21], and Patton and Crouch [22], respectively. Total sugars contents in the same supernatants desalted using a $10 \mathrm{kDa}$ membrane were determined according to the phenol sulphuric assay [23] and expressed as D-glucose equivalent (g.L $\left.{ }^{-1}\right)$.

\subsection{Exopolysaccharide production}

Strains identified as potential EPS producers were transferred to a modified $\mathrm{f} / 2$ medium supplemented in nitrogen and phosphorous sources $\left(1 \mathrm{~g}\right.$ of $\mathrm{NaNO}_{3}$ and $0.2 \mathrm{~g}$ of $\left.\mathrm{NaH}_{2} \mathrm{PO}_{4} .2 \mathrm{H}_{2} \mathrm{O}\right)$ and called enriched $\mathrm{f} / 2$ medium. These concentrations were determined based on a stoichiometric approach [24-25]. Strains were maintained photoautotrophically in $500 \mathrm{~mL}$ flasks containing $200 \mathrm{~mL}$ of enriched $\mathrm{f} / 2$ medium inoculated with $20 \mathrm{~mL}$ of microalgae culture in exponential phase of growth. Culture conditions were similar to those of screening. Strains were let growing in these conditions during 60 days in order to maximize the biomass production, including EPS.

\subsection{Exopolysaccharide extraction}

Cultures were centrifuged at $10000 \mathrm{~g}$ for $15 \mathrm{~min}$ at $20{ }^{\circ} \mathrm{C}$. EPS in supernatants were desalted by cycles of concentrations-dilutions (milliQ water) using an Amicon centrifugal filter equipped with a $10 \mathrm{kDa}$ NMWCO membrane. EPS were considered as purified when the conductivity of the filtrates was near to that of milliQ water (around $2 \mu{\mathrm{S} . \mathrm{cm}^{-1}}^{-1}$ ). Finally, EPS 
solutions were freeze-dried during $48 \mathrm{~h}$ and samples were stored at room temperature until further analyses.

\subsection{Monosaccharide compositions}

Monosaccharides compositions of EPS were both determined by High-Pressure Anion Exchange Chromatography equipped with Pulsed Amperometric Detection (HPAEC-PAD) and

Gas Chromatography coupled to Mass Spectrophotometer with Electron Ionization (GC/MSEI) to ensure data complementary. For both methods results were expressed in molar ratio percentages.

For HPAEC-PAD analyses, $10 \mathrm{mg}$ of EPS were dissolved in $1 \mathrm{~mL}$ of $2 \mathrm{M}$ trifluoroacetic acid (TFA) solution in a glass tube and heated at $120{ }^{\circ} \mathrm{C}$ for $90 \mathrm{~min}$. The samples were stirred 3 times during the hydrolysis. After hydrolysis, $\mathrm{pH}$ was adjusted to 7 by addition of ammonium hydroxide $(35 \% \mathrm{w} / \mathrm{v})$ and the solutions were centrifuged at $14000 \mathrm{~g}$ for $15 \mathrm{~min}$ at room temperature. Supernatants were filtered through $0.2 \mu \mathrm{m}$ membrane filter and analyzed by HPAEC with an ICS 3000 system (Dionex Corporation, Sunnyvale (CA), USA) equipped with pulsed amperometric detection (PAD) and AS 50 autosampler. Twenty-five $\mu \mathrm{L}$ of samples were injected in the system and eluted into a pre-column CarboPac PA1-column $(4 \times 50 \mathrm{~mm})$ and an analytical CarboPac PA1-column $(4 \times 250 \mathrm{~mm})$ equilibrated $15 \mathrm{~min}$ with $18 \mathrm{mM} \mathrm{NaOH}$. Samples were eluted isocratically with $18 \mathrm{mM} \mathrm{NaOH}$ for $25 \mathrm{~min}$, followed by a linear gradient between 0 to $0.5 \mathrm{M}$ sodium acetate in $200 \mathrm{mM} \mathrm{NaOH}$ for $20 \mathrm{~min}$ to elute acidic monosaccharides. Run was followed by 15 min washing with $200 \mathrm{mM} \mathrm{NaOH}$. The eluent flow rate was kept constant at $1 \mathrm{~mL} \cdot \mathrm{min}^{-1}$. Columns were thermostated at $25^{\circ} \mathrm{C}$. Data were collected and analyzed with Dionex Chromeleon 6.80 software (Sunnyvale, USA). L-Rha, D-Rib, L-Fuc, L-Ara, D-Xyl, D-Man, D-Gal, D-Glc, D- GlcA, and D-GalA were used as standards.

For GC/MS analyses, $15 \mathrm{mg}$ of EPS were dissolved in $1.5 \mathrm{~mL}$ of TFA in a glass tube followed by heating at $120{ }^{\circ} \mathrm{C}$ for $90 \mathrm{~min}$. The samples were mixed each $30 \mathrm{~min}$. The 
preparations were then evaporated under nitrogen stream. The dried residues were dissolved in BSTFA: TMCS (99: 1) following the procedure described by Pierre et al. [26-27], then evaporated again under nitrogen stream. Dichloromethane was finally added to the derivatized products prior to injection onto GC/MS-EI apparatus. Standards (L-Rha, D-Rib, L-Fuc, L-Ara, D-Xyl, D-Man, D-Gal, D-Glc, D-GlcA, D-GalA, D-GlcN, D-GalN) were prepared following the same procedure. Analyses were carried out by GC/MS-EI using an Agilent 6890 Series GC System coupled to an Agilent 5973 Network Mass Selective detector. Derivatives were analyzed using an OPTIMA-1MS (30 m, $0.32 \mathrm{~mm}, 0.25 \mathrm{~mm})$ column, under helium flow of 2.3 $\mathrm{mL} \cdot \mathrm{min}^{-1}$. The helium pressure was set to $8.8 \mathrm{psi}$ and the split ratio to 50:1. The temperature was programmed to $100{ }^{\circ} \mathrm{C}$ for $3 \mathrm{~min}$ then raised to $200^{\circ} \mathrm{C}$ at $8{ }^{\circ} \mathrm{C} \cdot \mathrm{min}^{-1}$ keeping for $1 \mathrm{~min}$ then continuing with a final temperature increment to $215{ }^{\circ} \mathrm{C}$ at $5{ }^{\circ} \mathrm{C} \cdot \mathrm{min}^{-1}$. The ionization was carried out by Electronic Impact (EI, $70 \mathrm{eV})$. The trap temperature was fixed to $150{ }^{\circ} \mathrm{C}$ and the target ion was from 40 to $800 \mathrm{~m} / \mathrm{z}$. Data processing was done with LC/MSD Trap Version 5.3 and MestreNova Version 7.1 Softwares (Agilent).

\subsection{Relationship between phylogeny and exopolysaccharide composition}

\subsubsection{Statistics analysis}

Differences in levels of each monosaccharide were tested between 5 of the 6 microalguae phyla. Miozoa were not included in the analysis, since only two monosaccharide compositions were available. For each monosaccharide, Kruskal-Wallis tests were performed. When a significant difference was found $(\mathrm{p}<0.05)$, this test was followed by multiple pairwise comparisons between phyla (Mann-Whitney tests), with significance levels adjusted according to the Benjamini and Hochberg method [28].

\subsubsection{Phylogenetic tree}

Monosaccharide compositions of 81 EPS from microalgae (including those from the strains CCAP 904/1, RCC4633, RCC3686, RCC6688, RCC3514, RCC1544, RCC1545, 
RCC4037, RCC4120, RCC3438, RCC3688, RCC3685, RCC3707, RCC2380, RCC1564, RCC133, RCC4435, RCC5, RCC2684 and RCC4628), belonging to 6 major phyla and 15 different classes, were compiled in a phylogenetic tree. The classification used was that of Algaebase database (http://www.algaebase.org). Algaebase is an on-line database providing free access to authoritative taxonomic, distributional and nomenclatural information [29]. The nucleotide sequences coding for $18 \mathrm{~S}$ ribosomal RNA (18S rRNA) were searched on the National Center for Biotechnology Information (NCBI) database for all these strains. For 41 species, 1 to 3 sequences were found on this database. For the others, sequences obtained from species belonging to the same genus were used. Other sequences have also been added to construct a phylogenetic tree consistent with recently published phylogenetic trees [30-32]. 214 sequences were obtained and aligned using the MUSCLE algorithm [33] available in the MEGA 7 software [34]. Then the tree was constructed using the Neighbor-Joining method with the MEGA 7 software over a total of 1248 positions, based on the p-distance method with 2000 bootsraps. All positions containing gaps and missing data were eliminated. The monosaccharide compositions (in $\%$ of the total composition or presence/absence) of 81 strains were added to this phylogenetic tree using the iTOL tool [35]. All classes were supported by bootstrap values higher than 0.92, except for Zygnematophyceae (0.55), Chlorophyceae (0.54) and Trebouxiophyceae $(0.35)$.

\section{Results and Discussion}

\subsection{Screening of strains with EPS+ phenotype}

Hundred and sixty-six (150 microalgae and 16 cyanobacteria) strains were selected from the 5852 strains of the RCC based on the following criteria: i) cover a maximum of phylogenetic diversity, ii) target classes and orders not described for the production of EPS, iii) select different species to study the diversity of compositions within the same genus and iv) choose strains with a "promising phenotype" as both biofilm and gel formations or slimy appearance 
231 during the cultures. Short morphologic descriptions of strains for which genus and species names are

232 not known are available on the Roscoff Culture Collection (http://roscoff-culture-collection.org/). To

233 identify positive strains two conditions were required: growing under the chosen conditions and

234 producing a minimum of 0.05 g.L.- $\mathrm{L}^{-1}$ of true EPS at D30 (30 days). These criteria were evaluated

235 thanks to the results of $A_{750}$, nitrate (or ammonium) and total carbohydrates assays (Table 1;

236 Supplementary data Figure S1). In support of these results, the 166 strains were classified

237 into three groups. 
Table 1 part 1: Growth $\left(\mathrm{A}_{750}\right)$, production of extracellular carbohydrates and phenotype of 166 microalgae and cyanobacteria from the RCC.

\begin{tabular}{|c|c|c|c|c|c|c|c|c|c|c|}
\hline Kingdoms & Phyla & Classes & Orders & Families & Genera & Species & RCC numbers & $\begin{array}{c}\text { Growth } \\
\left(\mathbf{A}_{750}\right)^{ \pm}\end{array}$ & $\begin{array}{c}\text { Dubois } \\
\text { assay [22] }\end{array}$ & Groups \\
\hline \multirow{16}{*}{ Bacteria } & \multirow{16}{*}{ Cyanobacteria } & \multirow{16}{*}{ Cyanophyceae } & Chroococcales & Microcystaceae & Microcystis & sp. & 26 & + & 0.00 & EPS- \\
\hline & & & Nostocales & Aphanizomenonaceae & Nodularia & spumigena & 4299 & + & 0.00 & EPS- \\
\hline & & & Pleurocapsales & Xenococcaceae & Xenococcus & sp. & 2703 & + & 0.00 & EPS- \\
\hline & & & Spirulinales & Spirulinaceae & Spirulina & sp. & 1786 & + & 0.00 & EPS- \\
\hline & & & \multirow{12}{*}{ Synechococcales } & \multirow{2}{*}{ Acaryochloridaceae } & \multirow{2}{*}{ Acaryochloris } & marina & 1983 & + & 0.00 & EPS- \\
\hline & & & & & & tomasi & 1774 & + & 0.00 & EPS- \\
\hline & & & & Leptolyngbyaceae & Leptolyngbya & sp. & 232 & + & 0.00 & EPS- \\
\hline & & & & Prochloraceae & Prochlorococcus & sp. & 269 & + & 0.00 & EPS- \\
\hline & & & & \multirow{8}{*}{ Synechococcaceae } & Cyanobium & sp. & 2436 & + & 0.00 & EPS- \\
\hline & & & & & \multirow{7}{*}{ Synechococcus } & \multirow{7}{*}{ sp. } & 2380 & + & $0.12 *$ & EPS+ \\
\hline & & & & & & & 1084 & + & 0.00 & EPS- \\
\hline & & & & & & & 2035 & + & 0.00 & EPS- \\
\hline & & & & & & & 2368 & + & 0.00 & EPS- \\
\hline & & & & & & & 2381 & + & 0.00 & EPS- \\
\hline & & & & & & & 2383 & + & 0.00 & EPS- \\
\hline & & & & & & & 4555 & + & 0.00 & EPS- \\
\hline
\end{tabular}

The classification was done according to the AlgaeBase database [29]. producer,

NGS: Not Growing-Strain, * value obtained at 150 and $\mu \mathrm{mol} \mathrm{m}{ }^{-2} \cdot \mathrm{s}^{-1}$ and $* *$ value obtained at $300 \mu \mathrm{mol} \mathrm{m}^{-2} \cdot \mathrm{s}^{-1},{ }^{ \pm}$growth expressed as significant increase of $\mathrm{A}_{750}$ in time. 
Table 1 part 2: Growth $\left(\mathrm{A}_{750}\right)$, production of extracellular carbohydrates and phenotype of 166 microalgae and cyanobacteria from the RCC.

\begin{tabular}{|c|c|c|c|c|c|c|c|c|c|c|}
\hline Kingdoms & Phyla & Classes & Orders & Families & Genera & Species & $\begin{array}{c}\text { RCC } \\
\text { numbers }\end{array}$ & $\begin{array}{c}\text { Growth } \\
\left(\mathrm{A}_{750}\right)^{ \pm}\end{array}$ & $\begin{array}{c}\text { Dubois } \\
\text { assay [22] }\end{array}$ & Groups \\
\hline & & & & & Cylindrotheca & sp. & 2608 & - & - & NGS \\
\hline & & & & & Nitzschia & sp. & 2638 & - & - & NGS \\
\hline & & 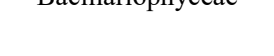 & Fragilariales & & & & 2598 & + & 0.00 & EPS- \\
\hline & & & Naviculales & Naviculaceae & Navicula & sp. & 3092 & - & - & NGS \\
\hline & & Chlorarachniophyceae & Chlorarachniales & Chlorarachniaceae & Bigelowiella & natans & 2352 & + & $0.05^{*}$ & EPS+ \\
\hline & & Coscinodiscophyceae & Rhizosoleniales & Rhizosoleniaceae & Guinardia & flaccida & 3093 & - & - & NGS \\
\hline & & \multirow{9}{*}{ Cryptophyceae } & \multirow{2}{*}{ Chromulinales } & Chromulinaceae & Ochromonas & sp. & 2350 & - & - & NGS \\
\hline & & & & Chrysamoebaceae & Chrysamoeba & sp. & 377 & - & - & NGS \\
\hline \multirow[t]{9}{*}{ Chromista } & \multirow[t]{9}{*}{ Ochrophyta } & & \multirow{3}{*}{ Cryptomonadales } & \multirow{3}{*}{ Hemiselmidaceae } & \multirow{2}{*}{ Chroomonas } & sp. & 1504 & - & - & NGS \\
\hline & & & & & & sp. & 3436 & + & 0.02 & EPS- \\
\hline & & & & & Hemiselmis & sp. & 2614 & - & - & NGS \\
\hline & & & \multirow{4}{*}{ Pyrenomonadales } & \multirow{2}{*}{ Geminigeraceae } & \multirow{2}{*}{ Proteomonas } & sp. & 3072 & + & 0.02 & EPS- \\
\hline & & & & & & sulcata & 3649 & - & - & NGS \\
\hline & & & & \multirow{2}{*}{ Pyrenomonadaceae } & \multirow{2}{*}{ Rhinomonas } & sp. & 821 & + & 0.00 & EPS- \\
\hline & & & & & & sp. & 1978 & + & 0.02 & EPS- \\
\hline & & \multirow{2}{*}{ Dictyochophyceae } & Florenciellales & & Pseudochattonella & verruculosa & 1082 & - & - & NGS \\
\hline & & & Rhizochromulinales & Rhizochromulinaceae & Rhizochromulina & sp. & 4438 & - & - & NGS \\
\hline
\end{tabular}

The classification was done according to the AlgaeBase database [29]. .

NGS: Not Growing-Strain, * value obtained at 150 and $\mu \mathrm{mol} \mathrm{m}-2 . \mathrm{s}^{-1}$ and $* *$ value obtained at $300 \mu \mathrm{mol} \mathrm{m}{ }^{-2} \cdot \mathrm{s}^{-1},{ }^{ \pm}$growth expressed as significant increase of $\mathrm{A}_{750}$ in time. 
Table 1 part 3: Growth $\left(\mathrm{A}_{750}\right)$, production of extracellular carbohydrates and phenotype of 166 microalgae and cyanobacteria from the RCC.

\begin{tabular}{|c|c|c|c|c|c|c|c|c|c|c|}
\hline Kingdoms & Phyla & Classes & Orders & Families & Genera & Species & $\begin{array}{c}\text { RCC } \\
\text { numbers }\end{array}$ & $\begin{array}{l}\text { Growth } \\
\left(\mathbf{A}_{750}\right)^{ \pm}\end{array}$ & $\begin{array}{c}\text { Dubois } \\
\text { assay [22] }\end{array}$ & Groups \\
\hline \multirow{17}{*}{ Chromista } & \multirow{17}{*}{ Ochrophyta } & Mediophyceae & Chaetocerotales & Chaetocerotaceae & Chaetoceros & sp. & 2564 & - & - & NGS \\
\hline & & \multirow{8}{*}{ Mediophyceae } & \multirow{2}{*}{ Cymatosirales } & \multirow{2}{*}{ Cymatosiraceae } & Papiliocellulus & sp. & 2624 & + & 0.00 & EPS- \\
\hline & & & & & Minutocellulus & sp. & 1863 & - & - & NGS \\
\hline & & & Eupodiscales & Eupodiscaceae & Odontella & sp. & 2558 & - & - & NGS \\
\hline & & & Lithodesmiales & Lithodesmiaceae & Ditylum & brightwellii & 775 & - & - & NGS \\
\hline & & & \multirow{4}{*}{ Thalassiosirales } & Skeletonemataceae & Skeletonema & sp. & 2932 & - & - & NGS \\
\hline & & & & \multirow{3}{*}{ Thalassiosiraceae } & Minidiscus & trioculatus & 4657 & - & - & NGS \\
\hline & & & & & \multirow{2}{*}{ Thalassiosira } & sp. & 1714 & - & - & NGS \\
\hline & & & & & & weissflogii & 76 & - & - & NGS \\
\hline & & \multirow{6}{*}{ Pelagophyceae } & \multirow{3}{*}{ Pelagomonadales } & \multirow{3}{*}{ Pelagomonadaceae } & \multirow{2}{*}{ Aureococcus } & \multirow{2}{*}{ anophagefferens } & 96 & - & - & NGS \\
\hline & & & & & & & 4094 & - & - & NGS \\
\hline & & & & & Pelagococcus & sp. & 3069 & + & $0.10 * *$ & EPS+ \\
\hline & & & \multirow{2}{*}{ Sarcinochrysidales } & \multirow{2}{*}{ Sarcinochrysidaceae } & Andersenia & nodulosa & 4631 & + & 0.01 & EPS- \\
\hline & & & & & Aureoumbra & lagunensis & 97 & - & - & NGS \\
\hline & & & & & & & 2696 & + & 0.01 & EPS- \\
\hline & & Phaeothamniophyceae & Aurearenales & Aurearenaceae & Aurearena & cruciata & 4621 & + & 0.01 & EPS- \\
\hline & & Pinguiophyceae & Pinguiochrysidales & Pinguiochrysidaceae & Glossomastix & sp. & 1196 & + & 0.01 & EPS- \\
\hline
\end{tabular}

The classification was done according to the AlgaeBase database [29]. = : w: no classification available. +: positive result, -: negative result, EPS+: positive strain, EPS producer, EPS-: negative strain, no EPS producer,

NGS: Not Growing-Strain, * value obtained at 150 and $\mu \mathrm{mol} \mathrm{m}-2 . \mathrm{s}^{-1}$ and $* *$ value obtained at $300 \mu \mathrm{mol} \mathrm{m}{ }^{-2} \cdot \mathrm{s}^{-1},{ }^{ \pm}$growth expressed as significant increase of $\mathrm{A}_{750}$ in time. 
Table 1 part 4: Growth $\left(\mathrm{A}_{750}\right)$, production of extracellular carbohydrates and phenotype of 166 microalgae and cyanobacteria from the RCC.

\begin{tabular}{|c|c|c|c|c|c|c|c|c|c|c|}
\hline Kingdoms & Phyla & Classes & Orders & Families & Genera & Species & $\begin{array}{c}\text { RCC } \\
\text { numbers }\end{array}$ & $\begin{array}{c}\text { Growth } \\
\left(\mathbf{A}_{750}\right)^{ \pm}\end{array}$ & $\begin{array}{c}\text { Dubois } \\
\text { assay [22] }\end{array}$ & Groups \\
\hline & & & & & & sp. & 3685 & + & $0.06^{*}$ & EPS+ \\
\hline & & & & & Glossomastix & sp. & 3688 & + & $0.10 * *$ & EPS+ \\
\hline & & 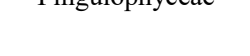 & 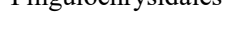 & 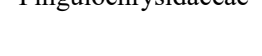 & & sp. & 3707 & + & $0.11^{*}$ & EPS+ \\
\hline & Ochrophyta & & & & Phaeomonas & sp. & 4470 & + & $0.07 * *$ & EPS+ \\
\hline & & & & & Fibrocapsa & japonica & 1501 & - & - & NGS \\
\hline & & Raphidophyceae & Chattonellales & Fibrocapsaceae & Heterosigma & akashino & 1502 & + & 0.01 & EPS- \\
\hline & & Synchromophyceae & Synchromales & Synchromaceae & Guanchochroma & wildpretii & 3390 & - & - & NGS \\
\hline & & & & & & & CC 19 & + & $0.13^{*}$ & EPS+ \\
\hline \multirow[t]{9}{*}{ Chromista } & \multirow{9}{*}{ Haptophyta } & \multirow{9}{*}{ Coccolithophyceae } & \multirow{9}{*}{ Coccolithales } & \multirow{2}{*}{ Calcidiscaceae } & Calcidiscus & leptoporus & 1170 & - & - & NGS \\
\hline & & & & & Oolithotus & fragilis & 2321 & - & - & NGS \\
\hline & & & & \multirow{2}{*}{ Calyptrosphaeraceae } & \multirow{2}{*}{ Calyptrosphaera } & sp. & 1180 & + & $0.77 * *$ & EPS+ \\
\hline & & & & & & sphaeroidea & 1178 & - & - & NGS \\
\hline & & & & Coccolithaceae & Coccolithus & braarudii & 3777 & - & - & NGS \\
\hline & & & & \multirow{4}{*}{ Hymenomonadaceae } & Hymenomonas & coronata & 1337 & + & $0.05^{*}$ & EPS+ \\
\hline & & & & & \multirow{3}{*}{ Ochrosphaera } & verrucosa & 3650 & + & $0.05^{*}$ & EPS+ \\
\hline & & & & & & \multirow{2}{*}{ sp. } & 3635 & + & $0.05^{*}$ & EPS+ \\
\hline & & & & & & & 2976 & - & - & NGS \\
\hline
\end{tabular}

The classification was done according to the AlgaeBase database [29]. "w: no classification available. +: positive result, -: negative result, EPS+: positive strain, EPS producer, EPS-: negative strain, no EPS producer, NGS: Not Growing-Strain, * value obtained at 150 and $\mu \mathrm{mol} \mathrm{m}{ }^{-2} . \mathrm{s}^{-1}$ and ${ }^{* *}$ value obtained at $300 \mu \mathrm{mol} \mathrm{m}{ }^{-2} \cdot \mathrm{s}^{-1},{ }^{ \pm}$growth expressed as significant increase of $\mathrm{A}_{750}$ in time. 
Table 1 part 5: Growth $\left(\mathrm{A}_{750}\right)$, production of extracellular carbohydrates and phenotype of 166 microalgae and cyanobacteria from the RCC.

\begin{tabular}{|c|c|c|c|c|c|c|c|c|c|c|}
\hline Kingdoms & Phyla & Classes & Orders & Families & Genera & Species & RCC numbers & $\begin{array}{c}\text { Growth } \\
\left(\mathbf{A}_{750}\right)^{ \pm}\end{array}$ & $\begin{array}{c}\text { Dubois } \\
\text { assay }[22]\end{array}$ & Groups \\
\hline \multirow{18}{*}{ Chromista } & \multirow{18}{*}{ Haptophyta } & \multirow{18}{*}{ Coccolithophyceae } & \multirow{3}{*}{ Isochrysidales } & \multirow{3}{*}{ Isochrysidaceae } & \multirow{3}{*}{ Chrysotila } & sp. & 4633 & + & $0.22 *$ & EPS+ \\
\hline & & & & & & sp. & CCAP 904/1 & + & $0.16^{*}$ & EPS+ \\
\hline & & & & & & roscoffensis & 1395 & + & 0.03 & EPS- \\
\hline & & & \multirow{6}{*}{ Isochrysidales } & \multirow{3}{*}{ Isochrysidaceae } & Isochrysis & braarudii & 3686 & + & $0.06^{*}$ & EPS+ \\
\hline & & & & & Ruttnera & lamellosa & 4061 & + & $0.05^{*}$ & EPS+ \\
\hline & & & & & Tisochrysis & lutea & 3699 & + & 0.02 & EPS- \\
\hline & & & & \multirow{3}{*}{ Noelaerhabdaceae } & \multirow{2}{*}{ Emiliania } & \multirow{2}{*}{ huxleyi } & 1216 & + & $0.07^{* *}$ & EPS+ \\
\hline & & & & & & & 1217 & + & 0.02 & EPS- \\
\hline & & & & & Gephyrocapsa & oceanica & 1314 & - & - & NGS \\
\hline & & & \multirow{8}{*}{ Phaeocystales } & \multirow{8}{*}{ Phaeocystaceae } & \multirow{2}{*}{ Phaeocystis } & \multirow{2}{*}{ globosa } & 739 & - & - & NGS \\
\hline & & & & & & & 1719 & - & - & NGS \\
\hline & & & & & \multirow{6}{*}{ Phaeocystis } & \multirow{6}{*}{ sp. } & 851 & - & - & NGS \\
\hline & & & & & & & 908 & - & - & NGS \\
\hline & & & & & & & 925 & - & - & NGS \\
\hline & & & & & & & 940 & - & - & NGS \\
\hline & & & & & & & 2055 & - & - & NGS \\
\hline & & & & & & & 3539 & - & - & NGS \\
\hline & & & Prymnesiales & Prymnesiaceae & Prymnesium & parvum & 3426 & + & $0.12^{*}$ & EPS+ \\
\hline
\end{tabular}

The classification was done according to the AlgaeBase database [29]. . no classification available. +: positive result, -: negative result, EPS+: positive strain, EPS producer, EPS-: negative strain, no EPS producer, NGS: Not Growing-Strain, * value obtained at 150 and $\mu \mathrm{mol} \mathrm{m}{ }^{-2} \cdot \mathrm{s}^{-1}$ and ${ }^{* *}$ value obtained at $300 \mu \mathrm{mol} \mathrm{m} \mathrm{m}^{-2} \cdot \mathrm{s}^{-1},{ }^{ \pm}$growth expressed as significant increase of $\mathrm{A}_{750}$ in time. 
Table 1 part 6: Growth $\left(\mathrm{A}_{750}\right)$, production of extracellular carbohydrates and phenotype of 166 microalgae and cyanobacteria from the RCC.

\begin{tabular}{|c|c|c|c|c|c|c|c|c|c|c|}
\hline Kingdoms & Phyla & Classes & Orders & Families & Genera & Species & $\begin{array}{c}\text { RCC } \\
\text { numbers }\end{array}$ & $\begin{array}{c}\text { Growth } \\
\left(\mathbf{A}_{750}\right)^{ \pm}\end{array}$ & $\begin{array}{c}\text { Dubois } \\
\text { assay }[22]\end{array}$ & Groups \\
\hline & & & & & Imantonia & rotunda & 1342 & + & 0.04 & EPS- \\
\hline & & & Prymnesiales & & Haptolina & hirta & 3421 & - & - & NGS \\
\hline & & Coccolithophyceae & & Chrysochromulinaceae & Chrysochromulina & camella & 1186 & - & - & NGS \\
\hline & & & Zygodiscales & Pontosphaeraceae & Scyphosphaera & apsteinii & 1456 & - & - & NGS \\
\hline & & & & & & & 3704 & + & $0.05^{*}$ & EPS+ \\
\hline & & \multirow{12}{*}{ Pavlovophyceae } & \multirow{12}{*}{ Pavlovales } & \multirow{12}{*}{ Pavlovaceae } & \multirow{3}{*}{ Diacronema } & sp. & 3514 & + & $0.29 * *$ & EPS+ \\
\hline & & & & & & lutheri & 1537 & + & 0.01 & EPS- \\
\hline & & & & & & viridis & 3459 & + & 0.02 & EPS- \\
\hline \multirow[t]{9}{*}{ Chromista } & \multirow[t]{9}{*}{ Haptophyta } & & & & \multirow{2}{*}{ Exanthemachrysis } & \multirow{2}{*}{ sp. } & 1536 & + & $0.06^{*}$ & EPS+ \\
\hline & & & & & & & 1544 & + & $0.10^{*}$ & EPS+ \\
\hline & & & & & \multirow{6}{*}{ Pavlova } & enorae & 1525 & + & $0.05^{*}$ & EPS+ \\
\hline & & & & & & gyrans & 1545 & + & $0.05^{*}$ & EPS+ \\
\hline & & & & & & \multirow{4}{*}{ sp. } & 4037 & + & $0.09 *$ & EPS+ \\
\hline & & & & & & & 4120 & + & $0.08^{*}$ & EPS+ \\
\hline & & & & & & & 4070 & + & $0.07^{*}$ & EPS+ \\
\hline & & & & & & & 3438 & + & $0.39 *$ & EPS+ \\
\hline & & & & & Rebecca & $\mathrm{sp}$. & 1528 & + & $0.05^{* *}$ & EPS+ \\
\hline
\end{tabular}

The classification was done according to the AlgaeBase database [29]. NGS: Not Growing-Strain, * value obtained at 150 and $\mu \mathrm{mol} \mathrm{m}{ }^{-2} \cdot \mathrm{s}^{-1}$ and ${ }^{* *}$ value obtained at $300 \mu \mathrm{mol} \mathrm{m} \mathrm{m}^{-2} \cdot \mathrm{s}^{-1}$, ${ }^{ \pm}$growth expressed as significant increase of $\mathrm{A}_{750}$ in time. 
Table 1 part 7: Growth $\left(\mathrm{A}_{750}\right)$, production of extracellular carbohydrates and phenotype of 166 microalgae and cyanobacteria from the RCC.

\begin{tabular}{|c|c|c|c|c|c|c|c|c|c|c|}
\hline Kingdoms & Phyla & Classes & Orders & Families & Genera & Species & $\begin{array}{c}\text { RCC } \\
\text { numbers }\end{array}$ & $\begin{array}{c}\text { Growth } \\
\left(\mathbf{A}_{750}\right)^{ \pm}\end{array}$ & $\begin{array}{c}\text { Dubois } \\
\text { assay [22] }\end{array}$ & Groups \\
\hline \multirow{16}{*}{ Chromista } & Haptophyta & & & & & & 6688 & + & $0.10^{*}$ & EPS+ \\
\hline & \multirow{15}{*}{ Miozoa } & \multirow{15}{*}{ Dinophyceae } & Gonyaulacales & Ostreopsidaceae & Alexandrium & minutum & 1490 & + & 0.01 & EPS- \\
\hline & & & \multirow{5}{*}{ Gymnodiniales } & \multirow{2}{*}{ Brachidiniaceae } & \multirow{2}{*}{ Karenia } & brevis & 4635 & - & - & NGS \\
\hline & & & & & & mikimotoi & 1513 & - & - & NGS \\
\hline & & & & \multirow{3}{*}{ Gymnodiniaceae } & Amphidinium & carterae & 1522 & + & $0.05^{* *}$ & EPS+ \\
\hline & & & & & Cochlodinium & polykrikoides & 4634 & - & - & NGS \\
\hline & & & & & Lepidodinium & chlorophorum & 1489 & - & - & NGS \\
\hline & & & \multirow{2}{*}{ Peridiniales } & \multirow{2}{*}{ Heterocapsaceae } & \multirow{2}{*}{ Heterocapsa } & \multirow{2}{*}{ sp. } & 3466 & + & 0.01 & EPS- \\
\hline & & & & & & & 2616 & - & - & NGS \\
\hline & & & Prorocentrales & Prorocentraceae & Prorocentrum & micans & 1517 & - & - & NGS \\
\hline & & & \multirow{4}{*}{ Suessiales } & \multirow{4}{*}{ Suessiaceae } & Biecheleriopsis & sp. & 3656 & - & - & NGS \\
\hline & & & & & Pelagodinium & sp. & 3003 & - & - & NGS \\
\hline & & & & & \multirow{2}{*}{ Symbiodinium } & kawagutii & 4019 & + & 0.02 & EPS- \\
\hline & & & & & & voratum & 1521 & + & 0.03 & EPS- \\
\hline & & & \multirow{2}{*}{ Thoracosphaerales } & \multirow{2}{*}{ Thoracosphaeraceae } & Thoracosphaera & heimii & 1512 & + & 0.01 & EPS- \\
\hline & & & & & Scrippsiella & trochoidea & 1515 & + & 0.01 & EPS- \\
\hline
\end{tabular}

The classification was done according to the AlgaeBase database [29].

NGS: Not Growing-Strain, * value obtained at 150 and $\mu \mathrm{mol} \mathrm{m}{ }^{-2} . \mathrm{s}^{-1}$ and** value obtained at $300 \mu \mathrm{mol} \mathrm{m}^{-2} . \mathrm{s}^{-1},{ }^{ \pm}$growth expressed as significant increase of $\mathrm{A}_{750}$ in time. 
Table 1 part 8: Growth $\left(\mathrm{A}_{750}\right)$, production of extracellular carbohydrates and phenotype of 166 microalgae and cyanobacteria from the RCC.

\begin{tabular}{|c|c|c|c|c|c|c|c|c|c|c|}
\hline Kingdoms & Phyla & Classes & Orders & Families & Genera & Species & $\begin{array}{c}\text { RCC } \\
\text { numbers }\end{array}$ & $\begin{array}{c}\text { Growth } \\
\left(\mathbf{A}_{750}\right)^{ \pm}\end{array}$ & $\begin{array}{c}\text { Dubois } \\
\text { assay [22] }\end{array}$ & Groups \\
\hline & & & & & & chui & 128 & + & $0.05^{*}$ & EPS + \\
\hline & & & & & & globosa & 1564 & + & $0.05^{*}$ & EPS+ \\
\hline & & & & & & rubens & 133 & + & $0.05^{*}$ & EPS+ \\
\hline & & Chlorodendrophyceae & Chlorodendrales & Chlorodendraceae & Tetraselmis & striata & 130 & + & 0.02 & EPS- \\
\hline & & & & & & & 4435 & + & $0.21 * *$ & EPS+ \\
\hline & & & & & & sp. & 119 & + & 0.03 & EPS- \\
\hline \multirow[t]{7}{*}{ Plantae } & Chlorophyta & & & Chlamydomonadaceae & Chlamydomonas & sp. & 3443 & + & 0.01 & EPS- \\
\hline & & Chlorophyceae & Chlamydomonadales & & & sp. & 5 & + & $0.06 * *$ & EPS+ \\
\hline & & & & Dürantracecac & Danamelia & tertiolecta & 6 & + & 0.02 & EPS- \\
\hline & & & & Bathycoccaceae & Bathycoccus & prasinos & 4222 & - & - & NGS \\
\hline & & Mamiellophyceae & Mamiellales & & Micromonas & commoda & 827 & + & 0.01 & EPS- \\
\hline & & & & & Ostreococcus & tauri & 1108 & + & 0.01 & EPS- \\
\hline & & Nephrophyceae & Nephroselmidales & Nephroselmidaceae & Nephroselmis & sp. & 1805 & + & 0.03 & EPS- \\
\hline
\end{tabular}

The classification was done according to the AlgaeBase database [29].

NGS: Not Growing-Strain, * value obtained at 150 and $\mu \mathrm{mol} \mathrm{m} \mathrm{m}^{-2} \cdot \mathrm{s}^{-1}$ and ${ }^{* *}$ value obtained at $300 \mu \mathrm{mol} \mathrm{m}{ }^{-2} \cdot \mathrm{s}^{-1},{ }^{ \pm}$growth expressed as significant increase of $\mathrm{A}_{750}$ in time. 
Table 1 part 9: Growth $\left(\mathrm{A}_{750}\right)$, production of extracellular carbohydrates and phenotype of 166 microalgae and cyanobacteria from the RCC.

\begin{tabular}{|c|c|c|c|c|c|c|c|c|c|c|}
\hline Kingdoms & Phyla & Classes & Orders & Families & Genera & Species & $\begin{array}{c}\text { RCC } \\
\text { numbers }\end{array}$ & $\begin{array}{c}\text { Growth } \\
\left(\mathbf{A}_{750}\right)^{ \pm}\end{array}$ & $\begin{array}{c}\text { Dubois } \\
\text { assay [22] }\end{array}$ & Groups \\
\hline & & & & & Prasinococcus & sp. & 2684 & + & $0.07 *$ & EPS+ \\
\hline & & 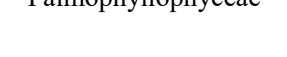 & Hasinutureares & 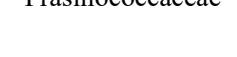 & Prasinoderma & sp. & 2686 & + & 0.02 & EPS- \\
\hline & & Picocystophyceae & Picocystales & Picocystaceae & Picocystis & salinarum & 3402 & + & 0.02 & EPS- \\
\hline & & & & & & & 2336 & + & 0.01 & EPS- \\
\hline & & Pyramimonadophyceae & Pseudoscourfieldiales & Pycnococcaceae & Pуспососсиs & provasolii & 3439 & + & 0.01 & EPS- \\
\hline & Chlorophyta & & Pyramimonadales & Pyramimonadaceae & Pyramimonas & sp. & 4212 & - & - & NGS \\
\hline & & & & & Chlorella & sp. & 2340 & + & $0.05^{*}$ & EPS+ \\
\hline \multirow[t]{8}{*}{ Plantae } & & & Chlorellales & Chlorellaceae & Nannochloris & sp. & 3644 & + & 0.00 & EPS- \\
\hline & & & & & Picochlorum & sp. & 3065 & + & 0.00 & EPS- \\
\hline & & & & Coccomyxaceae & Coccomyxa & sp. & 903 & + & $0.05^{*}$ & EPS+ \\
\hline & & & & & & & 3440 & - & - & NGS \\
\hline & \multirow{4}{*}{ Rhodophyta } & Porphyridiophyceae & Porphyridiales & Porphyridiaceae & Porphyridium & sp. & 4628 & + & $0.23 *$ & EPS+ \\
\hline & & Rhodellophyceae & Glaucosphaerales & Glaucosphaeraceae & Rhodella & maculata & 655 & + & $0.06 *$ & EPS+ \\
\hline & & \multirow{2}{*}{ Stylonematophyceae } & \multirow{2}{*}{ Stylonematales } & \multirow{2}{*}{ Stylonemataceae } & Chroodactylon & ramnosum & 654 & + & 0.01 & EPS- \\
\hline & & & & & Stylonema & sp. & 2964 & + & 0.00 & EPS- \\
\hline
\end{tabular}

The classification was done according to the AlgaeBase database [29]. NGS: Not Growing-Strain, * value obtained at 150 and $\mu \mathrm{mol} \mathrm{m}{ }^{-2} \cdot \mathrm{s}^{-1}$ and $* *$ value obtained at $300 \mu \mathrm{mol} \mathrm{m} \mathrm{m}^{-2} \cdot \mathrm{s}^{-1},{ }^{ \pm}$growth expressed as significant increase of $\mathrm{A}_{750}$ in time. 
Table 1 part 10: Growth $\left(\mathrm{A}_{750}\right)$, production of extracellular carbohydrates and phenotype of 166 microalgae and cyanobacteria from the RCC.

\begin{tabular}{|c|c|c|c|c|c|c|c|c|c|c|}
\hline Kingdoms & Phyla & Classes & Orders & Families & Genera & Species & RCC numbers & Growth $\left(\mathrm{A}_{750}\right)^{ \pm}$ & $\begin{array}{c}\text { Dubois assay } \\
{[22]}\end{array}$ & Groups \\
\hline & & & & & & & 3452 & + & $0.06 *$ & EPS+ \\
\hline & & & & & & & A13 880 & + & $0.08^{*}$ & EPS+ \\
\hline & & & & & & & CC 21 & + & $0.05^{*}$ & EPS+ \\
\hline & & & & & & & $\mathrm{CC} 76$ & + & $0.05^{*}$ & EPS+ \\
\hline & & & & & & & 3641 & + & 0.01 & EPS- \\
\hline & & & & & & & 4078 & + & 0.01 & EPS- \\
\hline & & & & & & & $18 / 1$ & + & 0.00 & EPS- \\
\hline & & & & & & & AST 713 & + & 0.01 & EPS- \\
\hline & & & & & & & CC 14 & + & 0.01 & EPS- \\
\hline & & & & & & & CC 20 & + & 0.02 & EPS- \\
\hline & & & & & & & 3448 & - & - & NGS \\
\hline & & & & & & & 3473 & - & - & NGS \\
\hline & & & & & & & AST 714 & - & - & NGS \\
\hline & & & & & & & JAP PR7 & - & - & NGS \\
\hline & & & & & & & JAP PR8 & - & - & NGS \\
\hline & & & & & & & KO 334 & - & - & NGS \\
\hline & & & & & & & OSH 51 & - & - & NGS \\
\hline
\end{tabular}

The classification was done according to the AlgaeBase database [29]. NGS: Not Growing-Strain, * value obtained at 150 and $\mu \mathrm{mol} \mathrm{m} \mathrm{m}^{-2} \cdot \mathrm{s}^{-1}$ and $\mathrm{d}^{* *}$ value obtained at $300 \mu \mathrm{mol} \mathrm{m} \mathrm{m}^{-2} \cdot \mathrm{s}^{-1},{ }^{ \pm}$growth expressed as significant increase of $\mathrm{A}_{750}$ in time. 
The first group (EPS + ) was composed of strains $(45 / 166)$ for which the total carbohydrates assays [23] gave a positive result for at least one of the two light intensities. The Table 1 gives the higher value of EPS production (obtained with an irradiance of 150 or 300 $\mu \mathrm{mol} \mathrm{m} \mathrm{m}^{-2} \cdot \mathrm{s}^{-1}$ ) for each strain. Some strains EPS+ produced EPS at the two irradiances (strains A13 880, CC19, CCAP904/1, RCC1180, RCC1544, RCC1545, RCC2684, RCC3426, RCC3438, RCC3514, RCC3685, RCC3688, RCC3707, RCC4037, RCC4120, RCC4435, RCC4470, RCC4628, RCC4633 and RCC6688) whereas some other strains were detected as EPS producers at only $150 \mu \mathrm{mol} \mathrm{m}{ }^{-2} \cdot \mathrm{s}^{-1}$ (CC21, CC76, RCC128, RCC133, RCC655, RCC903, RCC1337, RCC1525, RCC1536, RCC1564, RCC2340, RCC2352, RCC2380, RCC3452, RCC3635, RCC3650, RCC3686, RCC3704 and RCC4070) or $300 \mu \mathrm{mol} \mathrm{m} \mathrm{m}^{-2} \cdot \mathrm{s}^{-1}$ (RCC5, RCC1216, RCC1522, RCC1528 and RCC3069) irradiances. For the major part of these strains, the production of exocellular carbohydrates was correlated with a nitrogen starvation into the culture medium, as described in the literature [7]. This group included microalgae and cyanobacteria, together representing 7 phyla, 13 classes, 15 orders, 18 families and 24 genera. It was essentially composed of eukaryotes belonging to Chromista (30 species) or Plantae (10 species) kingdoms. Surprisingly, the Synechococcus sp., previously known for EPS production, was the sole member of prokaryotic domain whereas a large number of Cyanobateria has been described in previous studies for the production of EPS [36-37]. This observation could be explained by the very low number of screened cyanobacteria (only 16) but also by their ability to produce cell bound polysaccharides which are not measured in the extracellular medium. Four microalgae could not be phylogenetically classified due to a lack of information available in the RCC. The strains EPS+ from the kingdom Chromista belong mainly to the Haptophyta and Ochrophyta phyla. Haptophyta are essentially represented by the Coccolithophyceae and Pavlovophyceae classes. The family Isochrysidaceae was the most represented in Coccolithophyceae with $45 \%$ of EPS + strains. The family Pavlovaceae was the only 
representative of Pavlovophyceae. Among the Ochrophyta, the Pinguiochrysidaceae family included four strains out of five EPS+, ones belonging to the genera Phaeomonas and Glossomastix. The strains found EPS+ in the kingdom Plantae belong to the Chlorophyta and Rhodophyta phyla. In Chlorophyta, the family Chlorodendraceae was detached from others as it accounted for $40 \%$ of EPS+ strains. All these strains belong to the sole Tetraselmis genus. Regarding the Rhodophyta, two EPS+ strains belonging to the genera Rhodella and Porphyridium, which are well known in literature as EPS producers were detected [7].

The second group included strains not detected as EPS producers (EPS-) in the tested culture conditions. It was composed of 46 strains of microalgae and 15 strains of cyanobacteria. These strains are spread among 7 phyla, 20 classes, 29 orders, 34 families and 42 genera. Cell growth has been observed for all of them but not EPS production. For many microalgae strains, the release of EPS in the environment has been described to occur in response to a "stress", relating to a nutrient deficiency (nitrogen or phosphorous sources for instance), or the quality and the intensity of light or the salinity of the medium [38-44]. Nevertheless, the low amounts of nitrogen and phosphorous in the $\mathrm{f} / 2$ nutrient associated with growth cells observed by $\mathrm{A}_{750}$ measurements and the depletion of nitrogen (due to nitrate consumption) showed a nutrient deficiency, without production of EPS. Some strains of the group EPS- were able to grow to $300 \mu \mathrm{mol} \mathrm{m} \mathrm{m}^{-2} \cdot \mathrm{s}^{-1}$, suggesting that a high light intensity was not sufficient to induce an EPS production. Finally, other screening conditions may have prevented the EPS production, like inadequate $\mathrm{pH}$, temperature or agitation for example. Indeed, in nature benthic microalgae are less tolerant to shear stress than pelagic ones [45]. Stirring at $120 \mathrm{rpm}$ during the screening could affect the production of EPS for some strains. Another possible explanation would be the production of BPS, not measured by the methodology used during this work. Note also that despite their "promising phenotype" leading to their selection for this screening, these strains or a part of these strains could simply not be EPS producers. 
The third group corresponded to the strains for which no growth was observed during the screening, called "Not Growth-Strain" (NGS). It was composed of 60 strains distributed into 5 phyla, 13 classes, 28 orders, 35 families and 42 genera. The lack of growth could come from the composition of the culture medium due to the possible lack of some essential elements. For example, the silica is mandatory for the development of the Bacillariophyta (diatoms belonging to the Ochrophyta) [46]. This hypothesis was supported by the fact that most of diatoms fall into this group (except two, which were found EPS-). The salinity of the medium $\left(28\right.$ g.L $\left.\mathrm{L}^{-1}\right)$ can also be questioned.

Some strains belonging to families present in EPS- and NGS groups, including microalgae and cyanobacteria were also present in the EPS+ group such as Synechococcaceae (Cyanobacteria), Chlorodendraceae (Chlorophyta), Isochrysidaceae and Pavlovaceae (Haptophyta). However most of the microalgae and cyanobacteria present in these two groups belong to classes, and more specifically to genera that do not appear in the group of EPS+ strains. Table 2 presents an exhaustive list of strains identified in literature as EPS producers. Comparing the two data sets, most of them (20/25 total) belong to genera which were unknown for the production of soluble EPS. Additional analyzes should be needed to confirm the nature of extracellular sugars produced by EPS+ strains as EPS.

\subsection{Exopolysaccharide production and analysis}

Cultures of EPS+ strains were carried out for 60 days in order to produce EPS which were subsequently extracted. Then analyses by HPAEC-PAD and GC/MS-EI were performed to validate the positive results from the colorimetric assays. Analyses concerned 5 of the 7 phyla and 7 of the 15 orders of the EPS + strains ( $45 \%$ of the total strains). Some analyses were also performed on several EPS+ strains from the same family, and sometimes on different species belonging to the same genus in order to study the variability of the compositions. Table 3 shows the results obtained using each method. Despite few differences that can be observed between 
the two analytical methods, the results of the compositions were found to be similar, with the same main monosaccharides (Pearson: $\mathrm{R}^{2}=0.963 ; \mathrm{p}<0.001$ ). Among the 11 genera whose composition was analysed, 3 have been already described in literature, i.e. Synechococcus (RCC2380) [36], Dunaliella (RCC5) [43] and Porphyridium (RCC4628) [15, 47-49]. In this study the EPS from Dunaliella sp. (RCC5) was mainly composed of Gal, Rha, Xyl, Man and GalA $(28,21,17,13$ and $11 \%$, respectively) with some traces of other monosaccharides (Glc, Ara and GlcA). It presents some similarity with that previously described by Mishra and Jha [43] from D. salina which was composed of Xyl, Gal and Glc. 
Table 2, part 1: Monosaccharide and non sugars groups compositions of EPS from microalgae/cyanobacteria described in current literature.

\begin{tabular}{|c|c|c|c|c|c|c|c|c|c|c|c|c|c|c|c|}
\hline Genera (phyla) & Species & Rha & Gal & Ara & Glc & GlcA & GalA & Man & Fuc & Xyl & Rib & $\mathrm{SO}_{4}{ }^{2-}$ & Proteins & $\begin{array}{c}\text { Other } \\
\text { informations }\end{array}$ & References \\
\hline \multirow[t]{2}{*}{ Achnanthes (Oc) } & longipes & + & +++ & + & + & + & - & + & ++ & + & - & nd & $\mathrm{Nd}$ & & \multirow{2}{*}[58]{} \\
\hline & coffeaeformis & + & ++ & + & + & nd & nd & + & +++ & + & nd & nd & $\mathrm{Nd}$ & & \\
\hline \multirow[t]{2}{*}{ Amphora (Oc) } & holsatica & + & nd & + & + & nd & nd & nd & + & + & nd & nd & $\mathrm{Nd}$ & & {$[64]$} \\
\hline & rostrata & + & ++ & + & + & nd & nd & + & +++ & + & nd & + & + & pyruvate & {$[57]$} \\
\hline Ankistrodesmus (Chl) & densus & + & ++ & + & + & nd & nd & + & +++ & + & - & - & $\mathrm{Nd}$ & linkages & {$[66]$} \\
\hline Aulacoseira $(\mathrm{Oc})$ & granulata & +++ & + & nd & + & + & - & + & + & ++ & nd & nd & $\mathrm{Nd}$ & GlcNAc & {$[67]$} \\
\hline Botryococcus $(\mathrm{Chl})$ & braunii & + & +++ & + & $\operatorname{tr}$ & $\operatorname{tr}$ & - & $\operatorname{tr}$ & ++ & + & - & nd & + & & {$[68]$} \\
\hline \multirow[t]{2}{*}{ Bracteacoccus (Chl) } & $\mathrm{sp}$ & ++ & ++ & + & +++ & nd & nd & + & + & + & - & + & $\mathrm{Nd}$ & & {$[56]$} \\
\hline & affinis & + & + & + & - & nd & nd & - & + & - & nd & + & $\mathrm{Nd}$ & & {$[69]$} \\
\hline \multirow[t]{3}{*}{ Chaetoceros (Oc) } & curvisetus & + & ++ & - & - & nd & nd & - & +++ & - & - & + & $\mathrm{Nd}$ & linkages & [70] \\
\hline & decipiens & +++ & ++ & - & + & nd & nd & + & +++ & + & - & nd & $\mathrm{Nd}$ & linkages & [71] \\
\hline & agustae & - & + & + & +++ & ++ & - & + & - & + & - & nd & + & linkages & \multirow{2}{*}[72]{} \\
\hline \multirow[t]{2}{*}{ Chlamydomonas (Chl) } & corrosa & - & +++ & ++ & + & - & + & - & - & - & - & nd & + & linkages & \\
\hline & humicola & + & + & ++ & ++ & + & - & - & - & +++ & - & nd & + & & {$[73]$} \\
\hline
\end{tabular}

+++: first major monosaccharide, ++: 2nd major monosaccharide, +: presence, -: absence, tr: trace, nd: non determined. Cha: Charophyta, Chl: Chlorophyta, Mi: Miozoa, Oc: Ochrophyta, Rh: Rhodophyta. Rha: rhamnose, Gal: galactose, Ara: arabinose, Glc: glucose, Man: mannose, Fuc: fucose, Xyl: xylose, Rib: ribose, GlcA: glucuronic acid, GalA: galacturonic acid, GlcN: glucosamine, GalN: galactosamine, ManA: mannuronic acid, GlcNAc: $N$-acetyl-glucosamine, GalNAc: $N$-acetyl-galactosamine. 
Table 2, part 2: Monosaccharide and non sugars groups compositions of EPS from microalgae/cyanobacteria described in current literature.

\begin{tabular}{|c|c|c|c|c|c|c|c|c|c|c|c|c|c|c|c|}
\hline Genera (phyla) & Species & Rha & Gal & Ara & Glc & GlcA & GalA & Man & Fuc & Xyl & Rib & $\mathrm{SO}_{4}{ }^{2-}$ & Proteins & $\begin{array}{c}\text { Other } \\
\text { informations }\end{array}$ & References \\
\hline Chlamydomonas (Chl) & peterfii & - & +++ & + & + & - & ++ & - & - & - & - & nd & + & & {$[73]$} \\
\hline \multirow{6}{*}{ Chlamydomonas (Chl) } & reinhardtii & + & + & + & + & - & + & - & - & + & + & nd & - & pyruvate & {$[74]$} \\
\hline & mexicana & + & + & + & +++ & nd & nd & + & ++ & + & + & nd & $\mathrm{Nd}$ & & \multirow{2}{*}[75]{} \\
\hline & sajao & + & +++ & ++ & + & nd & nd & + & - & + & - & nd & $\mathrm{Nd}$ & & \\
\hline & sajao & - & +++ & + & + & - & ++ & - & - & - & - & nd & + & & {$[73]$} \\
\hline & stigmatophora & nd & nd & nd & nd & nd & nd & nd & nd & nd & nd & nd & $\mathrm{Nd}$ & & {$[76]$} \\
\hline & sp. & - & - & - & + & - & - & - & + & + & - & nd & $\mathrm{Nd}$ & & {$[77]$} \\
\hline \multirow{8}{*}{ Chlorella (Chl) } & autotrophica & nd & nd & nd & nd & nd & nd & nd & nd & nd & nd & nd & $\mathrm{Nd}$ & & {$[48]$} \\
\hline & mirabilis $(678 \mathrm{~F})$ & + & +++ & ++ & + & nd & nd & + & + & + & - & + & $\mathrm{Nd}$ & & \\
\hline & mirabilis $(7410 \mathrm{G})$ & + & +++ & + & + & nd & nd & + & ++ & + & - & $\operatorname{tr}$ & $\mathrm{Nd}$ & & {$[56]$} \\
\hline & ellipsoidea & + & +++ & + & + & nd & nd & ++ & + & + & + & + & $\mathrm{Nd}$ & & \\
\hline & pyrenoidosa & + & + & + & + & - & - & + & + & + & - & nd & $\mathrm{Nd}$ & & {$[78]$} \\
\hline & vulgaris & + & - & - & + & + & - & - & - & + & - & nd & $\mathrm{Nd}$ & & \multirow{3}{*}[77]{} \\
\hline & ellipsoidea & - & - & + & + & + & - & - & + & - & - & nd & $\mathrm{Nd}$ & & \\
\hline & sp. & + & - & + & + & + & - & - & - & - & - & nd & $\mathrm{Nd}$ & & \\
\hline Closterium (Cha) & sp. & + & + & + & + & +++ & - & + & ++ & + & - & nd & $\mathrm{Nd}$ & linkages & [54] \\
\hline Cochlodinium (Mi) & polykrikoides & - & + & - & + & + & + & + & - & - & - & + & - & & [79] \\
\hline
\end{tabular}


Table 2, part 3: Monosaccharide and non sugars groups compositions of EPS from microalgae/cyanobacteria described in current literature.

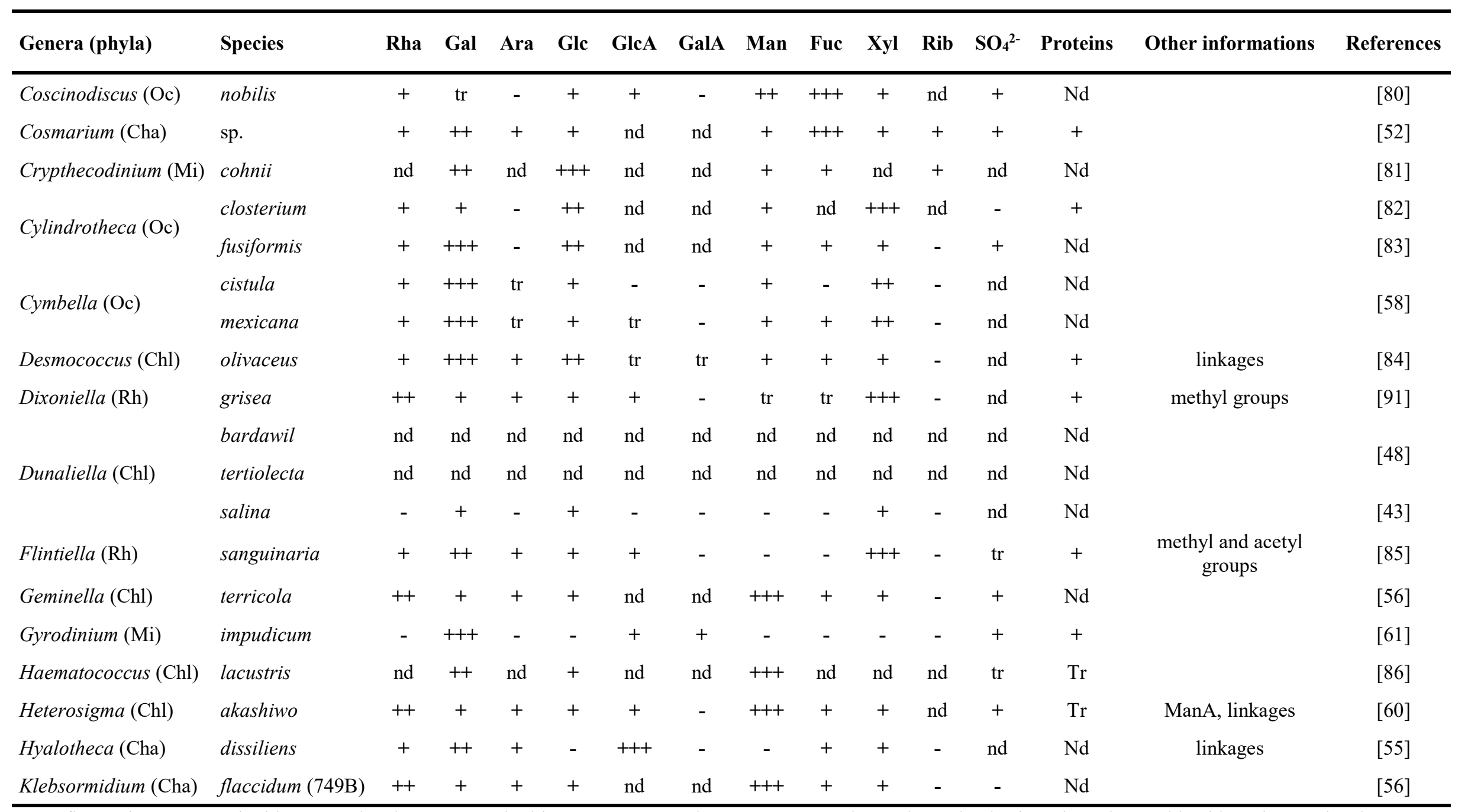

+++: first major monosaccharide, ++: 2nd major monosaccharide, +: presence, -: absence, tr: trace, nd: non determined. Cha: Charophyta, Chl: Chlorophyta, Mi: Miozoa, Oc: Ochrophyta, Rh: Rhodophyta. Rha: rhamnose, Gal: galactose, Ara: arabinose, Glc: glucose, Man: mannose, Fuc: fucose, Xyl: xylose, Rib: ribose, GlcA: glucuronic acid, GalA: galacturonic acid, GlcN: glucosamine, GalN: galactosamine, ManA: mannuronic acid, GlcNAc: $N$-acetyl-glucosamine, GalNAc: $N$-acetyl-galactosamine. 
Table 2, part 4: Monosaccharide and non sugars groups compositions of EPS from microalgae/cyanobacteria described in current literature.

\begin{tabular}{|c|c|c|c|c|c|c|c|c|c|c|c|c|c|c|c|}
\hline Genera (phyla) & Species & Rha & Gal & Ara & Glc & GIcA & GalA & Man & Fuc & Xyl & Rib & $\mathrm{SO}_{4}{ }^{2-}$ & Proteins & $\begin{array}{c}\text { Other } \\
\text { informations }\end{array}$ & $\begin{array}{c}\text { Referen } \\
\text { ces }\end{array}$ \\
\hline Klebsormidium (Cha) & flaccidum (446C) & +++ & + & + & + & nd & nd & ++ & + & + & - & $\operatorname{tr}$ & nd & & [56] \\
\hline Klebsormidium (Cha) & flaccidum (748A) & + & + & + & $\operatorname{tr}$ & nd & nd & +++ & ++ & + & - & + & nd & & {$[-0]$} \\
\hline Melosira $(\mathrm{Oc})$ & nummuloides & + & $\operatorname{tr}$ & + & + & nd & nd & nd & + & + & nd & nd & nd & & {$[65]$} \\
\hline \multirow[t]{2}{*}{ Micrasterias (Cha) } & denticulata & nd & nd & nd & nd & nd & nd & nd & nd & nd & nd & nd & nd & & {$[87]$} \\
\hline & directa & + & + & + & ++ & nd & nd & nd & +++ & + & nd & nd & nd & & {$[65]$} \\
\hline \multirow[t]{3}{*}{ Navicula (Oc) } & salinarum & + & + & - & +++ & nd & nd & + & nd & ++ & nd & + & + & & {$[82]$} \\
\hline & subinflata & + & ++ & + & +++ & nd & nd & + & + & + & + & + & + & pyruvate & {$[88]$} \\
\hline & digitus & + & + & + & + & nd & nd & + & +++ & ++ & $\operatorname{tr}$ & + & + & & \\
\hline \multirow[t]{2}{*}{ Netrium (Cha) } & interruptum & + & + & + & + & nd & nd & + & ++ & +++ & + & + & + & & {$[52]$} \\
\hline & oblongum & + & +++ & + & + & nd & nd & + & + & ++ & $\operatorname{tr}$ & + & + & & \\
\hline Oocystis (Chl) & sp. & - & + & + & - & + & - & - & + & - & - & nd & nd & & \multirow{2}{*}[77]{} \\
\hline Palmella (Chl) & mucosa & - & - & + & + & + & - & - & + & - & - & nd & nd & & \\
\hline \multirow{3}{*}{ Penium (Cha) } & cylindrus & + & + & + & + & nd & nd & + & +++ & ++ & $\operatorname{tr}$ & + & nd & & \multirow{2}{*}[52]{} \\
\hline & spirostriolatum & + & ++ & +++ & + & nd & nd & + & + & + & $\operatorname{tr}$ & + & + & & \\
\hline & margaritaceum & - & + & + & + & + & - & + & ++ & +++ & - & nd & nd & methyl groups & {$[89]$} \\
\hline Pleurotaenium (Cha) & trabecula & + & + & + & + & + & + & + & ++ & +++ & + & + & + & & [52] \\
\hline Porphyridium (Rh) & aerugineum & - & ++ & - & + & + & - & - & - & +++ & - & + & + & methyl groups & {$[50]$} \\
\hline
\end{tabular}


Table 2, part 5: Monosaccharide and non sugars groups compositions of EPS from microalgae/cyanobacteria described in current literature.

\begin{tabular}{|c|c|c|c|c|c|c|c|c|c|c|c|c|c|c|c|}
\hline Genera (phyla) & Species & Rha & Gal & Ara & Glc & GlcA & GalA & Man & Fuc & Xyl & Rib & $\mathrm{SO}_{4}{ }^{2-}$ & Proteins & Other informations & References \\
\hline \multirow{5}{*}{ Porphyridium (Rh) } & cruentum & + & ++ & + & + & nd & nd & + & + & +++ & - & + & + & \multirow{4}{*}{ methyl groups } & {$[47]$} \\
\hline & cruentum & - & + & - & ++ & + & - & - & - & +++ & - & + & + & & {$[50]$} \\
\hline & marinum & - & ++ & - & + & + & - & - & $\operatorname{tr}$ & +++ & - & + & nd & & [49] \\
\hline & purpureum & - & +++ & - & + & - & - & - & $\operatorname{tr}$ & ++ & - & + & - & & {$[15]$} \\
\hline & sp. & - & ++ & - & + & nd & nd & - & - & +++ & - & + & nd & \multirow[t]{2}{*}{ linkages } & {$[90]$} \\
\hline \multirow{3}{*}{ Rhodella (Rh) } & reticulata & + & + & - & ++ & + & - & + & - & +++ & + & nd & + & & {$[92]$} \\
\hline & maculata & + & +++ & + & + & + & - & - & - & ++ & - & + & + & \multirow{7}{*}{ linkages } & {$[15]$} \\
\hline & violacea & ++ & + & + & + & + & - & - & - & +++ & - & + & + & & {$[25]$} \\
\hline Scenedesmus (Chl) & quadricauda & nd & nd & nd & nd & nd & nd & nd & nd & nd & $\mathrm{Nd}$ & nd & nd & & {$[93]$} \\
\hline $\begin{array}{l}\text { Spondylosium } \\
\text { (Cha) }\end{array}$ & panduriforme & $\operatorname{tr}$ & ++ & + & + & + & - & - & ++ & + & - & - & + & & {$[53]$} \\
\hline Staurastrum (Cha) & iversenii & + & ++ & - & - & - & - & - & +++ & + & - & - & + & & {$[94]$} \\
\hline \multirow{2}{*}{ Stichococcus (Chl) } & $\begin{array}{l}\text { bacillaris (772B } \\
747 \mathrm{C})\end{array}$ & +++ & + & + & + & nd & nd & + & $\operatorname{tr}$ & ++ & - & $\operatorname{tr}$ & nd & & \multirow{2}{*}[56]{} \\
\hline & $\begin{array}{l}\text { bacillaris (774E } \\
677 \mathrm{~A})\end{array}$ & +++ & ++ & + & + & nd & nd & + & + & + & - & $\operatorname{tr}$ & nd & & \\
\hline Tetmemorus (Cha) & brebissonii & + & ++ & + & + & nd & nd & + & +++ & + & $\operatorname{Tr}$ & + & + & linkages & {$[52]$} \\
\hline Thalassiosira (Oc) & sp. & ++ & + & - & + & + & - & +++ & + & + & $\mathrm{Nd}$ & nd & + & $\begin{array}{c}\text { GlcNAc, GalNAc, } \\
\text { linkages }\end{array}$ & [95] \\
\hline
\end{tabular}

+++: first major monosaccharide, ++: 2nd major monosaccharide, +: presence, -: absence, tr: trace, nd: non determined. Cha: Charophyta, Chl: Chlorophyta, Mi: Miozoa, Oc: Ochrophyta, Rh: Rhodophyta. Rha: rhamnose, Gal: galactose, Ara: arabinose, Glc: glucose, Man: mannose, Fuc: fucose, Xyl: xylose, Rib: ribose, GlcA: glucuronic acid, GalA: galacturonic acid, GlcN: glucosamine, GalN: galactosamine, ManA: mannuronic acid, GlcNAc: $N$-acetyl-glucosamine, GalNAc: $N$-acetyl-galactosamine. 
Table 2, part 6: Monosaccharide and non sugars groups compositions of EPS from microalgae/cyanobacteria described in current literature.

\begin{tabular}{|c|c|c|c|c|c|c|c|c|c|c|c|c|c|c|c|}
\hline Genera & Species & Rha & Gal & Ara & Glc & GlcA & GalA & Man & Fuc & Xyl & Rib & $\mathrm{SO}_{4}{ }^{2-}$ & Proteins & Other informations & References \\
\hline \multirow{7}{*}{ Anabaena } & augstmalis & $\operatorname{tr}$ & + & - & +++ & - & ++ & + & + & - & - & nd & nd & GalN & {$[63]$} \\
\hline & cylindrica & $\operatorname{tr}$ & + & - & +++ & + & - & + & + & ++ & - & nd & nd & other uronic acid & {$[96]$} \\
\hline & flos-aquae & - & - & - & + & + & - & - & - & + & + & nd & nd & & {$[77]$} \\
\hline & sp. & nd & nd & nd & nd & nd & nd & nd & nd & nd & nd & nd & nd & & {$[97]$} \\
\hline & spiroides & ++ & + & nd & + & +++ & - & + & + & + & nd & nd & nd & GlcNAc, GalNAc & {$[67]$} \\
\hline & sphaerica & - & +++ & $\operatorname{tr}$ & + & + & + & + & - & - & - & nd & nd & & \multirow{2}{*}[98]{} \\
\hline & torulosa & ++ & + & + & + & + & + & - & + & +++ & - & nd & nd & GlcN & \\
\hline Anacystis & nidulans & nd & nd & nd & nd & nd & nd & nd & nd & nd & nd & nd & nd & & [99] \\
\hline Aphanocapsa & halophytia & + & + & - & ++ & - & - & + & +++ & + & - & + & + & & {$[100]$} \\
\hline Aphanothece & halophytica & - & + & + & +++ & + & - & + & ++ & - & - & nd & nd & linkages & {$[101]$} \\
\hline \multirow{3}{*}{ Arthrospira } & maxima & ++ & + & - & ++ & + & + & + & + & +++ & - & nd & nd & & {$[102]$} \\
\hline & platensis & + & +++ & $\operatorname{tr}$ & + & $\operatorname{tr}$ & + & $\operatorname{tr}$ & + & ++ & nd & $\operatorname{tr}$ & + & & {$[103]$} \\
\hline & platensis & +++ & + & nd & + & ++ & ++ & $\operatorname{tr}$ & nd & + & nd & + & nd & & {$[12]$} \\
\hline \multirow{2}{*}{ Calothrix } & pulvinata & + & + & + & +++ & nd & nd & + & + & + & - & $\operatorname{tr}$ & nd & & {$[56]$} \\
\hline & sp. & - & ++ & + & +++ & $\operatorname{tr}$ & + & + & + & + & - & nd & nd & GlcN & {$[63]$} \\
\hline Chlorogloeopsis & sp. & + & + & + & ++ & + & +++ & + & + & - & - & nd & nd & & {$[98]$} \\
\hline
\end{tabular}

+++: first major monosaccharide, ++: 2nd major monosaccharide, +: presence, -: absence, tr: trace, nd: non determined. Rha: rhamnose, Gal: galactose, Ara: arabinose, Glc: glucose, Man: mannose, Fuc: fucose, Xyl: xylose, Rib: ribose, GlcA: glucuronic acid, GalA: galacturonic acid, GlcN: glucosamine, GalN: galactosamine, ManA: mannuronic acid, GlcNAc: $N$-acetyl-glucosamine, GalNAc: $N$-acetyl-galactosamine. 
Table 2, part 7: Monosaccharide and non sugars groups compositions of EPS from microalgae/cyanobacteria described in current literature.

\begin{tabular}{|c|c|c|c|c|c|c|c|c|c|c|c|c|c|c|c|}
\hline Genera & Species & Rha & Gal & Ara & Glc & GlcA & GalA & Man & Fuc & Xyl & Rib & $\mathrm{SO}_{4}{ }^{2-}$ & Proteins & Other informations & References \\
\hline \multirow{2}{*}{ Chroococcus } & minutus & + & + & + & +++ & + & $\operatorname{tr}$ & ++ & + & + & - & nd & + & GlcN, methyl groups & {$[104]$} \\
\hline & submarinus & + & + & + & ++ & + & + & + & ++ & +++ & + & + & + & GlcN & {$[64]$} \\
\hline Cyanospira & capsulata & - & - & + & + & - & +++ & + & + & - & - & - & + & & {$[105]$} \\
\hline \multirow{2}{*}{ Cyanothece } & sp. & - & + & - & +++ & + & + & ++ & + & + & - & - & $\operatorname{tr}$ & & {$[106]$} \\
\hline & $\mathrm{sp}$. & - & - & - & +++ & - & - & - & - & ++ & + & + & + & & {$[62]$} \\
\hline Fischerella & muscicola & - & + & - & +++ & + & + & + & + & + & - & nd & nd & & {$[98]$} \\
\hline \multirow{2}{*}{ Gloeocapsa } & kuetzingigiana & ++ & + & +++ & +++ & + & ++ & nd & + & +++ & + & nd & nd & GalN, trace GlcN & {$[107]$} \\
\hline & sp. & nd & nd & nd & nd & nd & nd & nd & nd & nd & nd & nd & nd & & {$[13]$} \\
\hline Gloeocapsosis & crepidinum & + & + & + & ++ & + & + & nd & +++ & + & + & nd & nd & GlcN, GalN & {$[107]$} \\
\hline Gloeothece & sp. & + & ++ & - & +++ & nd & nd & + & - & + & - & + & + & methyl groups & {$[108]$} \\
\hline Johannesbaptistia & pellucida & + & + & + & + & + & + & + & + & + & - & + & + & & {$[64]$} \\
\hline \multirow{3}{*}{ Leptolyngbya } & foveolarum & ++ & + & - & +++ & nd & nd & + & - & + & - & nd & nd & & \multirow{2}{*}[56]{} \\
\hline & tenuis & ++ & + & - & +++ & nd & nd & + & + & + & - & $\operatorname{tr}$ & nd & & \\
\hline & sp. & + & $\operatorname{tr}$ & $\operatorname{tr}$ & +++ & + & ++ & nd & + & + & + & nd & nd & GalN, traces GlcN & {$[107]$} \\
\hline Lyngbya & conferviodes & $\operatorname{tr}$ & ++ & $\operatorname{tr}$ & +++ & + & nd & + & $\operatorname{tr}$ & $\operatorname{tr}$ & - & nd & nd & & [109] \\
\hline Microcoleus & vaginatus & + & ++ & + & +++ & + & + & + & + & + & - & nd & + & & [41] \\
\hline
\end{tabular}

+++: first major monosaccharide, ++: 2nd major monosaccharide, +: presence, -: absence, tr: trace, nd: non determined. Rha: rhamnose, Gal: galactose, Ara: arabinose, Glc: glucose, Man: mannose, Fuc: fucose, Xyl: xylose, Rib: ribose, GlcA: glucuronic acid, GalA: galacturonic acid, GlcN: glucosamine, GalN: galactosamine, ManA: mannuronic acid, GlcNAc: $N$-acetyl-glucosamine, GalNAc: $N$-acetyl-galactosamine. 
Table 2, part 8: Monosaccharide and non sugars groups compositions of EPS from microalgae/cyanobacteria described in current literature.

\begin{tabular}{|c|c|c|c|c|c|c|c|c|c|c|c|c|c|c|c|}
\hline Genera & Species & Rha & Gal & Ara & Glc & GlcA & GalA & Man & Fuc & Xyl & Rib & $\mathrm{SO}_{4}{ }^{2-}$ & Proteins & Other informations & References \\
\hline \multirow[t]{2}{*}{ Microcoleus } & vaginatus & + & + & + & ++ & + & + & +++ & + & + & - & nd & + & GlcNAc, linkages & {$[84]$} \\
\hline & aeruginosa & ++ & + & nd & + & - & + & + & + & +++ & nd & nd & nd & GlcNAc, GalNAc & {$[67]$} \\
\hline \multirow{8}{*}{ Microcystis } & aeruginosa & +++ & - & - & + & nd & nd & ++ & - & - & - & - & - & & \\
\hline & aeruginosa flos-aquae & - & - & - & +++ & nd & nd & ++ & - & - & - & - & - & & {$[110]$} \\
\hline & viridis & ++ & - & - & +++ & nd & nd & + & + & - & - & nd & nd & & \\
\hline & calcicola & + & + & + & +++ & + & + & + & + & ++ & - & nd & + & & {$[73]$} \\
\hline & carneum & - & - & - & - & - & - & +++ & - & ++ & - & - & + & & {$[62]$} \\
\hline & commune & - & ++ & - & +++ & - & nd & + & - & - & nd & nd & nd & & \multirow{2}{*}[111]{} \\
\hline & flagelliforme & - & ++ & + & + & + & nd & $\operatorname{tr}$ & + & +++ & nd & nd & nd & & \\
\hline & sp. & - & + & + & + & - & - & + & - & - & - & nd & nd & & {$[77]$} \\
\hline \multirow[t]{6}{*}{ Nostoc } & sp. & + & ++ & - & +++ & + & + & + & - & + & - & nd & + & & {$[40]$} \\
\hline & insulare & - & - & ++ & +++ & + & - & - & - & - & - & - & $\operatorname{tr}$ & trace acetate, linkages & {$[112]$} \\
\hline & insulare & $\operatorname{tr}$ & $\operatorname{tr}$ & + & +++ & ++ & + & + & + & $\operatorname{tr}$ & - & nd & + & & {$[104]$} \\
\hline & muscoru & nd & nd & nd & nd & nd & nd & nd & nd & nd & nd & nd & nd & & \multirow{2}{*}[13]{} \\
\hline & entophytum & nd & nd & nd & nd & nd & nd & nd & nd & nd & nd & nd & nd & & \\
\hline & sp. & + & + & + & +++ & + & + & + & + & ++ & - & nd & nd & & {$[63]$} \\
\hline Oscillatoria & amphibia & + & + & $\operatorname{tr}$ & +++ & nd & nd & ++ & $\operatorname{tr}$ & + & - & nd & nd & & [109] \\
\hline
\end{tabular}


Table 2, part 9: Monosaccharide and non sugars groups compositions of EPS from microalgae/cyanobacteria described in current literature.

\begin{tabular}{|c|c|c|c|c|c|c|c|c|c|c|c|c|c|c|c|}
\hline Genera & Species & Rha & Gal & Ara & Glc & GIcA & GalA & Man & Fuc & Xyl & Rib & $\mathrm{SO}_{4}{ }^{2-}$ & Proteins & $\begin{array}{c}\text { Other } \\
\text { informations }\end{array}$ & References \\
\hline \multirow{4}{*}{ Oscillatoria } & corallinae & + & + & + & +++ & + & nd & ++ & + & + & - & nd & nd & \multirow{17}{*}{ other uronic acid } & [109] \\
\hline & sp. & $\operatorname{tr}$ & - & - & +++ & - & - & - & $\operatorname{tr}$ & $\operatorname{tr}$ & - & - & + & & {$[64]$} \\
\hline & sp. & - & + & + & ++ & + & +++ & - & + & + & - & nd & nd & & {$[98]$} \\
\hline & sp. & - & - & - & +++ & - & - & - & - & ++ & + & - & + & & {$[62]$} \\
\hline \multirow{13}{*}{ Phormidium } & autumnale & +++ & $\operatorname{tr}$ & - & + & - & + & + & $\operatorname{tr}$ & $\operatorname{tr}$ & + & nd & nd & & {$[63]$} \\
\hline & battersii & - & ++ & + & +++ & + & + & ++ & + & + & - & + & + & & {$[64]$} \\
\hline & ambiguum & +++ & ++ & + & ++ & + & + & + & + & + & - & $\operatorname{tr}$ & nd & & \multirow{5}{*}[56]{} \\
\hline & corium (442D \& 746B) & ++ & + & + & +++ & nd & nd & + & + & + & - & $\operatorname{tr}$ & nd & & \\
\hline & corium (444A) & + & +++ & + & ++ & nd & nd & + & + & + & - & $\operatorname{tr}$ & nd & & \\
\hline & corium $(674 \mathrm{~A})$ & ++ & + & - & + & nd & nd & + & - & +++ & - & nd & nd & & \\
\hline & corium $(743 \mathrm{D})$ & + & + & - & +++ & nd & nd & ++ & - & + & - & nd & nd & & \\
\hline & ectocarpi $(\mathrm{K} 5)$ & + & ++ & $\operatorname{tr}$ & +++ & + & nd & + & $\operatorname{tr}$ & + & - & nd & nd & & \multirow{6}{*}[109]{} \\
\hline & ectocarpi (ME3) & + & + & $\operatorname{tr}$ & +++ & + & nd & ++ & $\operatorname{tr}$ & + & - & nd & nd & & \\
\hline & ectocarpi (N182; C86) & - & + & - & +++ & + & nd & ++ & - & + & - & nd & nd & & \\
\hline & foveolarum (C52) & + & + & $\operatorname{tr}$ & +++ & + & nd & ++ & $\operatorname{tr}$ & + & - & nd & nd & & \\
\hline & foveolarum (MEU) & + & ++ & + & +++ & - & - & + & + & + & - & nd & nd & & \\
\hline & minutum (D5) & $\operatorname{tr}$ & +++ & - & ++ & nd & nd & + & + & + & - & nd & nd & & \\
\hline
\end{tabular}


Table 2, part 10: Monosaccharide and non sugars groups compositions of EPS from microalgae/cyanobacteria described in current literature.

\begin{tabular}{|c|c|c|c|c|c|c|c|c|c|c|c|c|c|c|c|}
\hline Genera & Species & Rha & Gal & Ara & Glc & GlcA & GalA & Man & Fuc & Xyl & Rib & $\mathrm{SO}_{4}{ }^{2-}$ & Proteins & $\begin{array}{c}\text { Other } \\
\text { informations }\end{array}$ & References \\
\hline \multirow{6}{*}{ Phormidium } & minutum (NB5) & $\operatorname{tr}$ & + & + & +++ & nd & nd & ++ & - & + & - & nd & nd & & \multirow{4}{*}{ [109] } \\
\hline & minutum (RT6) & + & + & ++ & +++ & + & nd & + & + & + & - & nd & nd & & \\
\hline & $\begin{array}{l}\text { sp. (CCAP1464/3 } \\
\text { CCAP1463/4) }\end{array}$ & + & ++ & + & +++ & + & nd & + & $\operatorname{tr}$ & + & - & nd & nd & & \\
\hline & sp. (PNG91；90-14/1) & + & + & + & +++ & nd & nd & ++ & + & + & - & nd & nd & & \\
\hline & sp. & + & + & + & +++ & + & + & - & + & ++ & - & nd & nd & & {$[98]$} \\
\hline & tепие & + & + & +++ & ++ & nd & $\operatorname{tr}$ & + & + & + & - & nd & + & $\begin{array}{l}\text { GlcNAc, } \\
\text { linkages }\end{array}$ & {$[84]$} \\
\hline \multirow{3}{*}{ Plectonema } & usteri & + & +++ & + & ++ & nd & nd & + & + & + & - & $\operatorname{tr}$ & nd & & {$[56]$} \\
\hline & golenkinianum & - & - & - & +++ & - & - & - & $\operatorname{tr}$ & $\operatorname{tr}$ & - & - & + & \multirow[b]{2}{*}{$\begin{array}{c}\text { GleN, trace } \\
\text { GalN }\end{array}$} & {$[64]$} \\
\hline & sp. & + & ++ & nd & +++ & $\operatorname{tr}$ & + & nd & $\operatorname{tr}$ & + & + & nd & nd & & {$[107]$} \\
\hline Rhabdoderma & rubrum & - & + & - & + & $\operatorname{tr}$ & $\operatorname{tr}$ & + & + & ++ & - & + & + & $\mathrm{GlcN}+++$ & {$[64]$} \\
\hline \multirow{2}{*}{ Scytonema } & hofmanni & - & + & - & + & + & + & +++ & - & - & - & nd & nd & \multirow{3}{*}{ linkages } & {$[98]$} \\
\hline & javanicum & + & ++ & + & +++ & $\operatorname{tr}$ & $\operatorname{tr}$ & + & $\operatorname{tr}$ & + & - & nd & + & & {$[84]$} \\
\hline Synechococcus & $\mathrm{sp}$. & nd & nd & nd & nd & nd & nd & nd & nd & nd & nd & nd & nd & & {$[36]$} \\
\hline \multirow{4}{*}{ Synechocystis } & aquatilis & + & + & - & +++ & + & + & + & + & + & - & nd & nd & $\mathrm{GlcN}++$ & {$[63]$} \\
\hline & sp. & nd & nd & nd & nd & nd & nd & nd & nd & nd & nd & nd & nd & & {$[13]$} \\
\hline & sp. (PCC6803) & + & + & - & +++ & + & + & + & ++ & + & - & + & + & GlcN, GalN & \multirow{2}{*}[113]{} \\
\hline & sp. (PCC6714) & + & ++ & + & +++ & + & + & ++ & + & + & - & + & + & GlcN, GalN & \\
\hline Tolypothrix & tenuis & + & + & + & +++ & + & + & ++ & + & - & - & nd & nd & GlcN & {$[98]$} \\
\hline \multirow{2}{*}{ Trichormus } & variabilis (VRUC162) & $\operatorname{tr}$ & + & $\operatorname{tr}$ & +++ & - & - & + & + & ++ & + & nd & nd & GalN & \multirow{2}{*}[63]{} \\
\hline & variabilis (VRUC168) & + & + & + & +++ & + & + & + & + & ++ & - & nd & nd & $\mathrm{GlcN}$ & \\
\hline
\end{tabular}

+++: first major monosaccharide, ++: 2nd major monosaccharide, +: presence, -: absence, tr: trace, nd: non determined. Rha: rhamnose, Gal: galactose, Ara: arabinose, Glc: glucose, Man: mannose, Fuc: fucose, Xyl: xylose, Rib: ribose, GlcA: glucuronic acid, GalA: galacturonic acid, GlcN: glucosamine, GalN: galactosamine, ManA: mannuronic acid, GlcNAc: $N$-acetyl-glucosamine, GalNAc: $N$-acetyl-galactosamine. 
Table 3: Monosaccharides compositions (molar ratios) of EPS from strains EPS+ obtained by GC/MS and HPAEC-PAD.

\begin{tabular}{|c|c|c|c|c|c|c|c|c|c|c|c|c|c|c|c|c|c|c|c|c|c|c|}
\hline \multirow{2}{*}{ Genera } & \multirow{2}{*}{ Species } & \multirow{2}{*}{ RCC numbers } & \multicolumn{2}{|c|}{ Rha } & \multicolumn{2}{|c|}{ Gal } & \multicolumn{2}{|c|}{ Ara } & \multicolumn{2}{|c|}{ Glc } & \multicolumn{2}{|c|}{ Man } & \multicolumn{2}{|c|}{ Fuc } & \multicolumn{2}{|c|}{ Xyl } & \multicolumn{2}{|c|}{ Rib } & \multicolumn{2}{|c|}{ GlcA } & \multicolumn{2}{|c|}{ GalA } \\
\hline & & & (1) & (2) & (1) & (2) & (1) & (2) & (1) & (2) & (1) & (2) & (1) & (2) & (1) & (2) & (1) & (2) & (1) & (2) & (1) & (2) \\
\hline \multirow{2}{*}{ Chrysotila } & dentata & CCAP 904/1 & - & - & 33 & 36 & 36 & 33 & $\operatorname{tr}$ & $\operatorname{tr}$ & 10 & 8 & - & $\operatorname{tr}$ & 17 & 16 & - & - & 4 & 4 & - & $\operatorname{tr}$ \\
\hline & sp. & 4633 & $\operatorname{tr}$ & $\operatorname{tr}$ & 31 & 33 & 13 & 11 & 42 & 45 & - & - & - & - & 10 & 8 & - & - & 4 & 2 & - & $\operatorname{tr}$ \\
\hline \multirow[t]{2}{*}{ Isochrysis } & braarudii & 3686 & 9 & 4 & 27 & 23 & 39 & 47 & 3 & 3 & - & - & - & - & 18 & 19 & + & + & 4 & 2 & - & - \\
\hline & & 6688 & 15 & 19 & 36 & 46 & 4 & $\operatorname{tr}$ & - & - & 34 & 32 & - & - & - & - & - & - & 11 & $\operatorname{tr}$ & - & $\operatorname{tr}$ \\
\hline Diacronema & sp. & 3514 & 33 & 37 & 26 & 23 & 17 & 8 & 15 & 16 & - & - & - & - & 5 & 10 & - & - & 4 & 4 & - & $\operatorname{tr}$ \\
\hline Exanthemachrysis & sp. & 1544 & 2 & 3 & 16 & 14 & 8 & 14 & 8 & 7 & 15 & 13 & - & - & 14 & 12 & - & - & 37 & 32 & - & 5 \\
\hline \multirow{4}{*}{ Pavlova } & gyrans & 1545 & 3 & 4 & 32 & 21 & 13 & 18 & 14 & 7 & - & - & $\operatorname{tr}$ & 6 & 21 & 25 & - & - & 9 & 11 & 8 & 7 \\
\hline & sp. & 4037 & 46 & 36 & 10 & 11 & 26 & 23 & 6 & 10 & - & - & - & 2 & 12 & 14 & - & - & - & 2 & - & 2 \\
\hline & sp. & 4120 & 7 & 7 & 34 & 40 & 13 & 4 & 23 & 35 & 4 & - & - & 7 & 19 & 2 & - & - & $\operatorname{tr}$ & 3 & - & 2 \\
\hline & sp. & 3438 & 47 & 30 & 27 & 29 & 5 & 14 & 11 & 17 & $\operatorname{tr}$ & - & - & - & 3 & 6 & - & - & 5 & 4 & - & - \\
\hline \multirow{3}{*}{ Glossomastix } & sp. & 3688 & 28 & 20 & 4 & 2 & - & - & - & - & - & - & 54 & 57 & - & - & - & - & 9 & 10 & 5 & 11 \\
\hline & sp. & 3685 & 20 & 19 & - & - & - & - & - & - & - & - & 56 & 57 & - & - & - & - & 10 & 8 & 14 & 13 \\
\hline & sp. & 3707 & 31 & 27 & 2 & 3 & - & - & - & $\operatorname{tr}$ & - & - & 40 & 47 & - & $\operatorname{tr}$ & - & - & 6 & 7 & 21 & 15 \\
\hline \multirow[t]{2}{*}{ Synechococcus } & sp. & 2380 & - & $\operatorname{tr}$ & 38 & 27 & - & $\operatorname{tr}$ & 38 & 49 & - & - & 24 & 20 & - & - & - & - & - & $\operatorname{tr}$ & - & $\operatorname{tr}$ \\
\hline & globosa & 1564 & 63 & 61 & 19 & 20 & $\operatorname{tr}$ & $\operatorname{tr}$ & 5 & 5 & - & - & - & - & 5 & 4 & - & - & - & 2 & 5 & - \\
\hline \multirow[t]{2}{*}{ Tetraselmis } & rubens & 133 & nd & 6 & $\mathrm{nd}$ & 89 & nd & - & nd & $\operatorname{tr}$ & nd & - & nd & 2 & nd & $\operatorname{tr}$ & nd & - & nd & - & nd & 2 \\
\hline & sp. & 4435 & 5 & 3 & 33 & 35 & 24 & 19 & 5 & 7 & 8 & 7 & - & 9 & 23 & 14 & - & - & 2 & 4 & - & 2 \\
\hline Dunaliella & sp. & 5 & 18 & 21 & 19 & 28 & 4 & 5 & 2 & $\operatorname{tr}$ & 11 & 13 & - & - & 13 & 17 & - & - & 19 & 4 & 14 & 11 \\
\hline Prasinococcus & sp. & 2684 & 7 & 4 & 67 & 74 & 7 & 3 & 9 & 7 & - & - & - & 2 & 7 & 9 & - & - & 3 & $\operatorname{tr}$ & - & - \\
\hline Porphyridium & sp. & 4628 & - & - & 29 & 27 & 2 & 2 & 22 & 20 & - & - & - & - & 44 & 49 & - & - & 3 & 2 & - & - \\
\hline
\end{tabular}

(1): GC/MS analyses, (2): HPAEC-PAD analyses, +: presence, -: absence, tr: trace, nd: non determined, 菐: no classification available. The SD was < to $5 \%$ for all experiments. 
EPS from Porphyridium sp. (RCC4628) had also a composition very similar to those already determined in the literature on different species of Porphyridium including aeruginum, marinum, cruentum and purpureum $[\mathbf{1 5}, \mathbf{4 7}, \mathbf{4 9 - 5 0 ]}$. It was a heteroxylan composed of Xyl, Gal and Glc (49, 27 and $20 \%$, respectively) and other minor monosaccharides. Phlips et al. [36] studied the physico-chemical properties of solutions of an EPS from a Synechococcus strain for which no monosaccharides composition was determined. Results obtained in this study revealed that the EPS from Synechococcus sp. (RCC2380) was composed of Glc, Gal and Fuc (49, 27 and $20 \%$, respectively).

This study also described for the first time the composition of EPS i) in species belonging to the Haptophyta phylum (Chrysotila (CCAP904/1, RCC4633), Diacronema (RCC3514), Exanthemachrysis (RCC1544), Isochrysis (RCC3686), Pavlova (RCC1545, RCC3438, RCC4120, RCC4037), ii) in two classes belonging to the Chlorophyta (Chlorodendrophyceae represented by the genus Tetraselmis (RCC133, RCC1564 and RCC4435) and Palmophyllophyceae with Prasinococcus sp. (RCC2684)), and iii) in the genus Glossomastix (RCC3685, RCC3688 and RCC3707) (Pinguiophyceae, Ochrophyta phylum). The compositions were extremely variable even if all EPS were heteropolysaccharides. They are mainly composed of three or five monosaccharides including neutral sugars and uronic acids apart for the biopolymers produced by Pavlova sp. (RCC4120) and Tetraselmis globosa (RCC1564) mainly composed of two monosaccharides representing 81 and $75 \%$ of the total monosaccharides, respectively. EPS of Tetraselmis rubens (RCC133) and Prasinococcus sp. (RCC2684) are galactans since Gal represents 74 and $89 \%$ of the total monosaccharides compositions, respectively. Table 3 shows that all the neutral sugars usually detected in the composition of microalgae/cyanobacteria EPS (Fuc, Ara, Rha, Gal, Glc, Gal, Xyl and Man) were present in the composition of biopolymers analyzed in this study. After analysis of the 17 EPS compositions, Gal, Rha, Xyl, Fuc, Ara, Glc and Man were respectively identified as the main 
monosaccharide in 7, 4, 2, 2, 1, 0 and 0 EPS (meaning for example Gal was the main monosaccharide in 7 EPS compositions). Similarly, Gal, Rha, Xyl, Fuc, Ara, Glc and Man were quantitatively the second monosaccharide in 6, 2, 2, 0, 3, 1 and 1 EPS compositions. The EPS of Exanthemachrysis sp. (RCC1544) was distinguished by its richness in GlcA representing around $35 \%$ of its composition. Note that PS mainly composed of glucuronic acids and called glucuronans are poorly detected in natural biomass and are very attractive for their biological and rheological properties [51]. In addition, most of the EPS identified in this study (with the exception of Synechococcus sp. (RCC2380)) contained at least one uronic acid (GlcA or GalA) and most of the time both. They are present in variable content, sometimes in trace amounts (EPS from RCC6688), in small quantities (EPS from Tetraselmis sp. (RCC4435) and Pavlova sp. (RCC4120 and RCC4037)) or in very large quantities as previously described in EPS from Exanthemachrysis sp. (RCC1544) and to a lesser degree in that of Pavlova gyrans with $35 \%$ and $11 \%$ of GlcA, respectively. Overall, this work confirmed that EPS from microalgae have very complex compositions, probably evolved in response to biotic and/or abiotic conditions.

\subsection{Relationship between phylogeny and exopolysaccharides compositions}

We performed a metanalysis on the EPS compositions of 81 strains of microalgae from bibliographic data and experimental results obtained in this study in order to determine whether they could vary according to their taxonomic affiliation. This metanalysis was not done on the composition of EPS from cyanobacteria as their specificity is the systematic presence of Glc as main $(63 \%)$ or second monosaccharides (18\%) (Table 2). Levels of some monosaccharides varied significantly between phyla $(\mathrm{p}<0.05$ in Kruskal-Wallis tests for Gal, Ara, Man, Fuc, Xyl, Rib and Uronic acids) while Rha and Glc levels were relativement constant across phyla

(Figure 1, $\mathrm{p}=0.117$ and $\mathrm{p}=0.058$, respectively). Such differences appear also on the phylogenetic tree presented on Figure 2. 


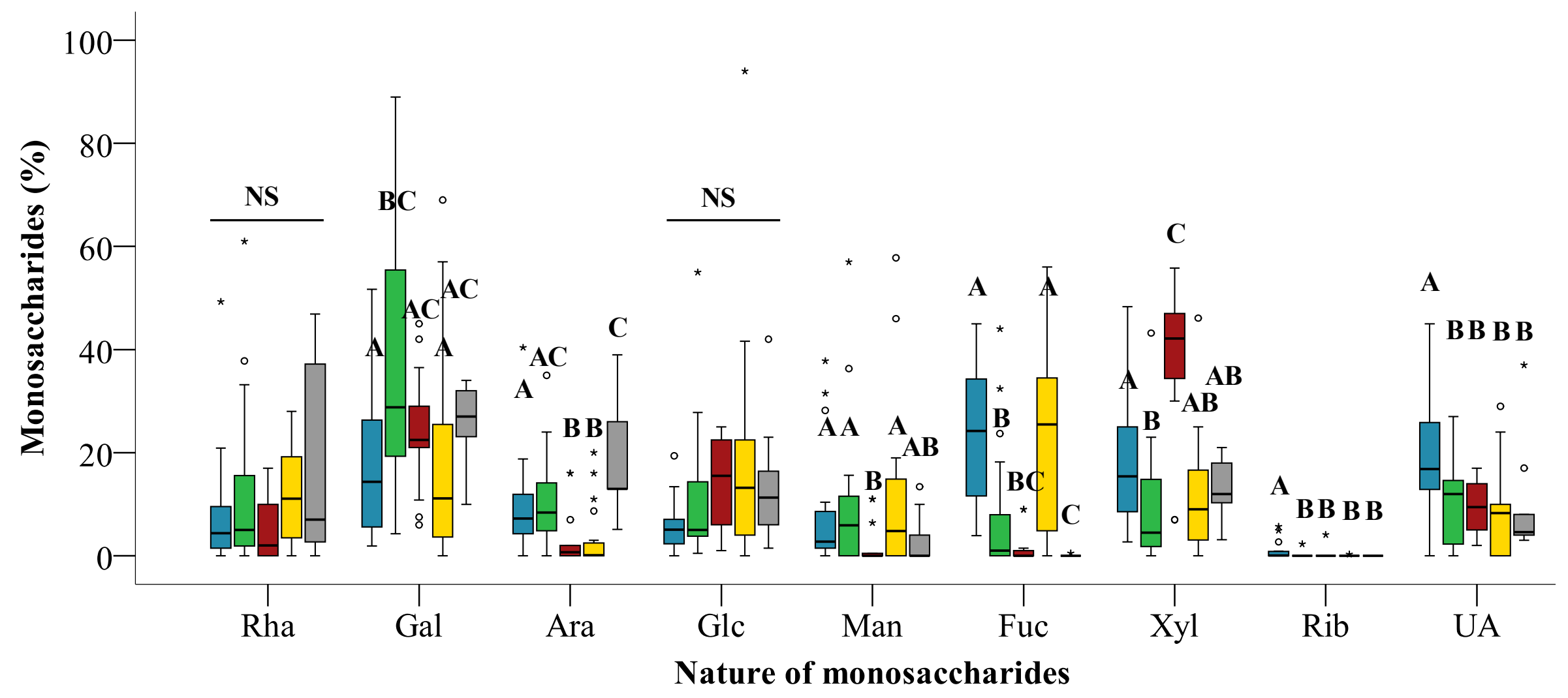

Figure 1: Variations in monosaccharides (Rha (rhamnose), Gal (galactose), Ara (arabinose), Glc (glucose), Man (mannose), Fuc (fucose), Xyl (xylose), Rib (ribose)) and uronic acids (UA), given in \% of the total composition) in EPS of Charophyta (匹), Chlorophyta (匹), Rhodophyta

Ochrophyta (-) and Haptophyta (-) (16,23, 13, 20 and 9 monosaccharides compositions, respectively). Data obtained from the literature and those included in this study are shown. Miozoa were not included in this analysis, since only two monosaccharide compositions were available. Miozoa were not included in this analysis, since only two monosaccharides compositions were available. Results are given as box-plots such as the central 
lines represent the medians, boxes the lower $25 \%$ to the upper $75 \%$ quartile, and the whiskers the range excluding outliers, that are given as open circles and stars. For each monosaccharide, Kruskal-Wallis tests were performed. When a significant difference was found $(\mathrm{p}<0.05)$, this test was followed by multiple pairwise comparisons (Mann-Whitney tests), with significance levels adjusted according to the Benjamini and Hochberg method (1995) [28]. Different letters indicate statistically significant differences between phyla. NS: Non-Significant results. The classification was done according to the AlgaeBase database [29]. Different letters indicate statistically significant differences between phyla. $\circ$ and $\star$ : outliers. NS: Non-Significant. 


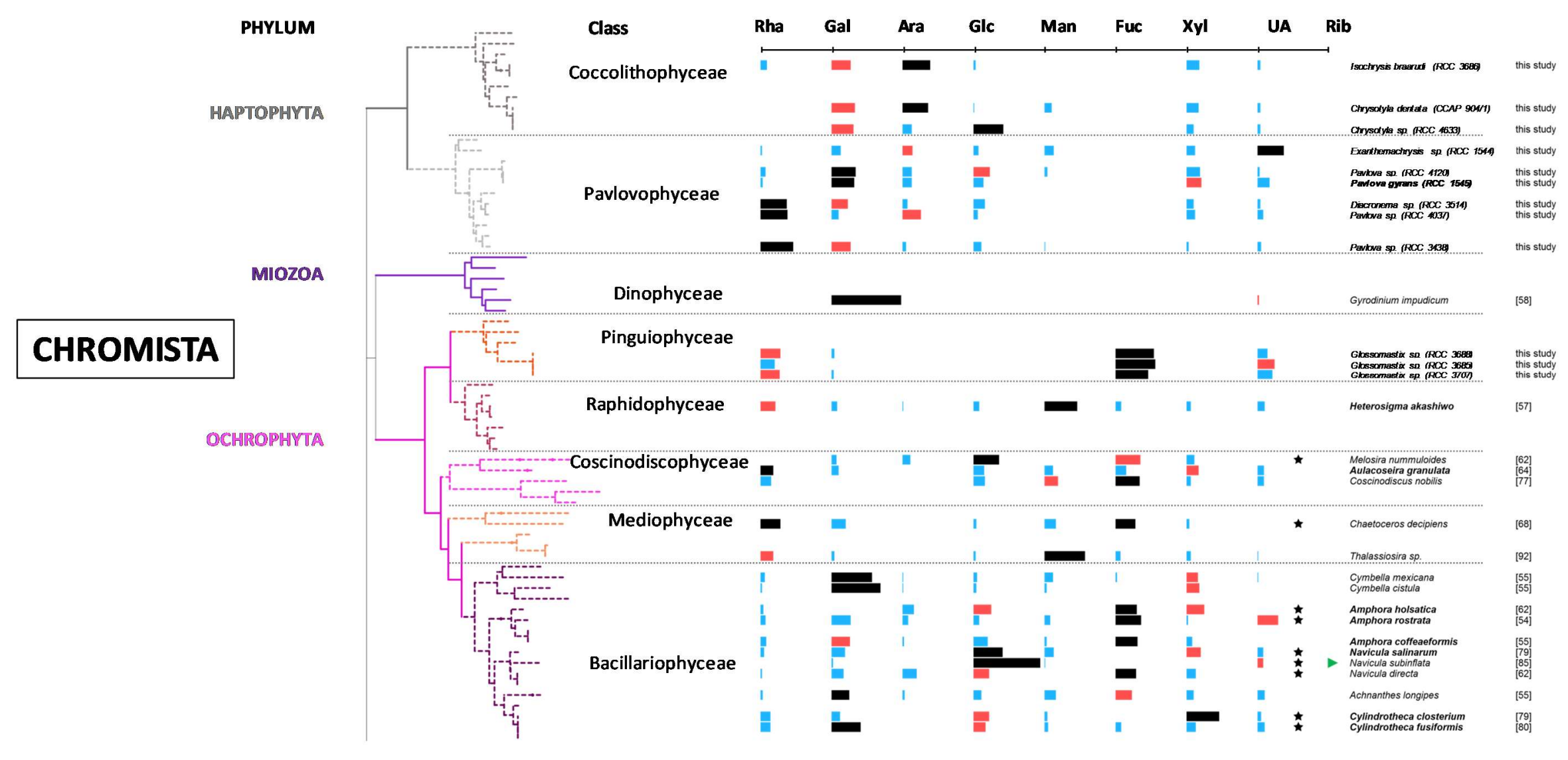




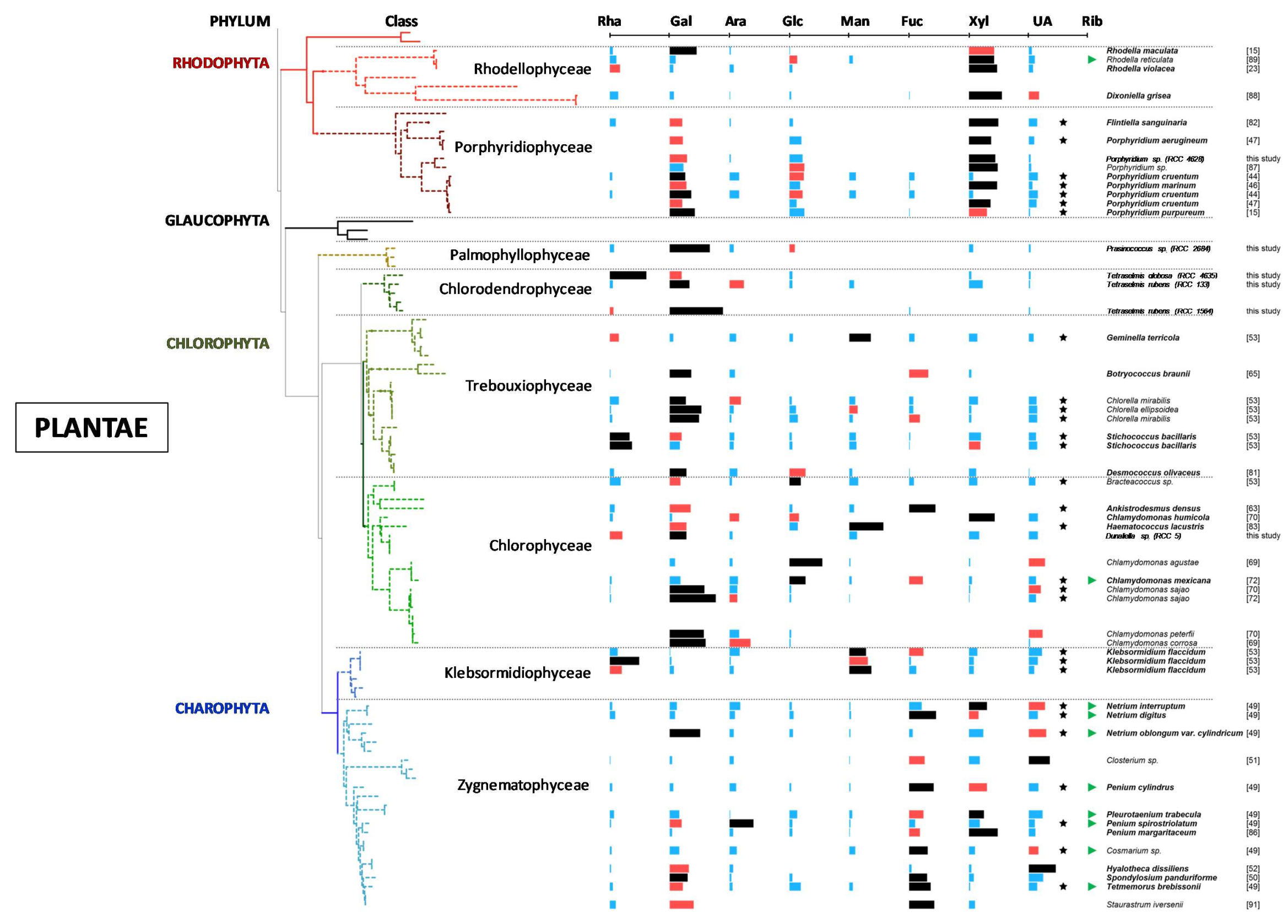


Figure 2, parts 1 and 2: Composition in neutral sugars (Rha (rhamnose), Gal (galactose), Ara (arabinose), Glc (glucose), Man (mannose), Fuc (fucose), Xyl (xylose), Rib (ribose)) and uronic acids (UA) (in \% of the total composition 0-100\% scale) of EPS from 81 microalgae (16 Charophyta, 23 Chlorophyta, 13 Rhodophyta, 20 Ochrophyta and 9 Haptophyta) placed on a Neighbor-Joining phylogenetic tree based on $18 \mathrm{~S}$ rDNA sequences. $\mathbf{m}: 1^{\text {st }}$ major monosaccharide, $\mathbf{m}: 2^{\text {nd }}$ major monosaccharide, $\mathbf{m}$ : secondary monosaccharides, $\star$ : \% of UA was obtained by colorimetric assay, : presence of Rib. The positions of the strains written in bold were determined from sequences corresponding to the right species. For the other species, sequences from a representative of the same genus were used. The classification was done according to the AlgaeBase database [29]. 
Clear differences, between the 3 phyla of the plantae kingdom included in the analysis (Charophyta, Chlorophyta and Rhodophyta), have been detected. EPS from Charophya have the most distinct composition profile, with higher Fuc levels compared to Chlorophyta and Rhodophyta. Fuc was the first or second monosaccharide in 10/16 profiles, while it was only $4 / 23$ profiles of the Chlorophyta and none in the Rhodophyta ( $p<0.001$ for each comparison). They also contain significantly higher levels of uronic acids compared to EPS from the two other Plantae phyla $(p=0.013$ and $p=0.01$ when compared to Chlorophyta and Rhodophyta, respectively). They can represent up to 28 and $29 \%$ in EPS of Netrium interruptum and Netrium oblongum, respectively [52]. In EPS from Spondylosium panduriform, uronic acids were only represented by GlcA which represents $24 \%$ of the total composition [53], and it is even the major monosaccharide in EPS from Closterium sp. and Hyalotheca dissiliens [54-55]. EPS from Charophya were also characterized by the presence of Rib ( $8 / 16$ profiles) which contrasts with those of Chlorophyta and Rhodophyta profiles (1/23 and $1 / 13$, respectively). This characteristic seems to be specific of the Zygnematophyceae (8/13 profiles) since Rib was not found in Klebsormidiophyceae. More specifically, it is systematically present in traces in Netrium, Cosmarium, Penium, Tetmemorus, Pleurotaenium genus [52]. A large majority of Charophyta EPS contain sulphate groups, except EPS from Klebsormidium flaccidum (749B) [56]. The sulphate content of some EPS can reach up $>10 \%$ as for those of Tetmemorus brebissonii and Pleurotaenium trabecula [52].

EPS from Rhodophya are characterized by high levels of Xyl $(p=0.005$ and $p=0.001$ when compared to Charophyta and Chlorophyta, respectively). This monosaccharide is the first or second in 11/13 profiles, which contrasts with those from Chlorophyta (2/23) and Charophyta (5/16). The Porphyridiaceae familly (including Porphyridium and Flintiella genera) was characterized by the production of galactoxylans, whereas EPS from the Glaucosphaeraceae 
familly were rhamnoxylans and/or galactoxylans, depending on the species. All these EPS were sulphated (excepted for Flintiella sanguinaria), sometimes with a content $>10 \%$ [49-50].

High levels of Gal were often observed in EPS from Chlorophyta (this monosaccharide being the first or second in $18 / 23$ profiles) which differs from Charophyta $(p=0.025)$ but not from Rhodophyta $(p=0.15)$, which have also Gal as a first or second monosaccharide in $9 / 13$ profiles.

The second kingdom analysed is the Chromista. Results presented part 3.2 have highlighted Haptophyta producing EPS. Thus, there are 3 phyla involved in EPS production, including Ochrophyta, Haptophyta, and Miozoa phyla. As only two monosaccharide compositions were available for Miozoa, comparaison analyses were only performed on Ochrophyta and Haptophyta. The main differences between Ochrophyta and Haptophyta lie in the differences in Fuc contents $(\mathrm{p}<0.001)$ (Figure 2). Indeed, Fuc is present in most EPS of Ochrophyta (17/20 profiles), as the first or second monosaccharide (11/20 profiles) whereas it is present in only 2 species of Haptophyta on the 10 analyzed. In the genera Amphora and Glossomastix in particular, Fuc represents respectively more than 30 and $46 \%$ of the total monosaccharide composition [57-58]. Differences in Ara levels can also be observed $(\mathrm{p}<$ 0.001). While most of the Ochrophyta EPS contain, at best, traces of Ara (content $>3 \%$ of the composition in only $4 / 20$ profiles), this monosaccharide is present at significantly higher levels in Haptophyta (content $>7 \%$ in $8 / 10$ profiles).

Although the analysis was focused on the distinctions of EPS compositions between phyla, it was contested that in the Chromista some species belonging to the same genus had very varied compositions, as Pavlova species for example. These differences could be explained by the fact that Pavlova is a polyphyletic genus [59]. Specific composition was found for the EPS of Heterosigma akashiwo which was the sole example of microalgae EPS containing 
ManA [60]. The composition of Gyrodinium impudicum EPS is also special because this is a homopolysaccharide: a sulphated galactan [61].

Finally, the last kingdom and also phylum is the cyanobacteria. EPS production by these photosynthetic microorganisms has been abundantly published, maybe because of their ecological abundance and easy growing. All these data make it possible to view the large diversity of composition of the exopolymers resulting from this phylum (Table 2). The main characteristic of cyanobacteria is the systematic presence of Glc, with the exception of Nostoc carneum [62]. It is the principal or second monosaccharide in $65(50 / 78$ profils) and $18 \%$ (14/78 profiles) EPS, respectively. In comparison, Xyl appearing as main monosaccharide just after Glc is 7 times less present (7/78 profiles). Some specific compositions have been highlighted. Osamines (GlcN and or GalN) were found in 21 compositions. In addition, GlcN is the first and second monosaccharide in EPS of Rhabdoderma rubrum and Synechocystis aquatilis, respectively [63-64]. Moreover, similar EPS compositions were found between strains belonging to the same genus, as Synechocystis [98, 113], Phormidium [109], Oscillatoria [64, 98, 109], Leptolyngbya [56, 107] and Plectonema [56, 64, 107].

\section{Conclusions}

One hundred and sixty-six microalgae and cyanobacteria were screened and 45 of them were detected as producers of a soluble EPS based on colorimetric assays. The composition analyses performed on 20 of them confirmed they were EPS. This allowed to highlight 8 new genera of microalgae and to characterize 17 new monosaccharide compositions. Overall, all the microalgae and cyanobacteria EPS are heteropolymers. They are produced by 49 and 29 genera in microalgae and cyanobacteria, respectively, which represent 134 species. Some common points in composition can be established according to the phylogenetic affiliation.

EPS from Charophya contain high Fuc and uronic acids levels and were characterized by the presence of Rib in the half part on EPS compositions available. Moreover, a large 
majority of their EPS contain sulphate groups. EPS from Rhodophya were characterized by high levels of Xyl, and the Porphyridiaceae and Glaucosphaeraceae families produce compositions that were specific to them. High levels of Gal were often observed in EPS from Chlorophyta. In Chromista, Fuc is present in most EPS of Ochrophyta compare to Haptophyta but Ara levels were significantly higher in Haptophyta compare to EPS of Ochrophyta. To conclude, EPS produced by cyanobacteria were mainly composed Glc, and this monosaccharide is always present.

Thanks to the growing number of studies and the 132 compositions available, the metanalysis on the EPS compositions by statistical tests made it possible to distinguish specific compositions between phyla. This finding is clearly the proof that evolution has conserved some complex biosynthesis pathways inside specific phyla to give an advantage to strains having the ability to produce some exopolysaccharides in some environments. However, the current lack of knowledge on the physiological function of EPS despite the abundance of literature on this subject makes it difficult to correlate some polysaccharidic structures with their biological roles. Nevertheless, there are still a lot of microalgae to study and only a tiny part of the phylogenetic tree has been concerned (insufficient data on Miozoa phylum for example).

\section{Conflict of interest}

The authors declare that they have no conflict of interest.

\section{Authors' contributions}

Philippe Michaud conceived the project and reviewed the manuscript. Céline Laroche designed the experiments for microalgae screening and culture in PBR; Guillaume Pierre, Cedric Delattre and Pascal Dubessay have setup methods for sugars analysis and molecular biology. Clément Gaignard has done the screening of microalgae and polysaccharides analysis, interpreted the results and wrote the manuscript. Christine Gardarin realised analysis by ionic chromatography; 
Aurore Dubuffet conducted the phylogenic analysis and interpreted the results; Priscillia Gourvil and Ian Probert have chosen the microalgae strains considering their phylogeny and phenotypes. All authors approved the final manuscript.

\section{Acknowledgment}

This work was supported by the Agence Nationale de la Recherche POLYSALGUE project, grant ANR-15-CE21-0013 of the French Agence Nationale de la Recherche.

\section{Statement of informed consent, human/animal rights}

No informed consent, human or animal rights applicable

\section{References}

[1] Ebenezer, V., Medlin, L.K., Ki, J.S., 2012. Molecular detection, quantification, and diversity evaluation of microalgae. Marine Biotechnology. 14, 129-142.

[2] Borowitzka, M.A., 2013. High-value products from microalgae - their development and commercialization. Journal of Applied Phycology. 25, 743-756.

[3] Gaignard, C., Gargouch, N., Dubessay, P., Delattre, C., Pierre, G., Laroche, C., Fendri, I., Abdelkafi, S., Michaud, P. New horizons in culture and valorization of red microalgae. Biotechnology Advances. 37, 193-222.

[4] Molina Grima, E., Belarbi, E.H., Acién Fernández, F.G., Robles Medina, A., Chisti, Y., 2003. Recovery of microalgal biomass and metabolites: process options and economics. Biotechnology Advances. 20, 491-515.

[5] Norsker, N.H., Barbosa, M.J., Vermuë, M.H., Wijffels, R.H., 2011. Microalgal production

- A close look at the economics. Biotechnology Advances. 29, 24-27. 
[6] Rossi, F., De Philippis, R., 2016. Exocellular polysaccharides in microalgae and cyanobacteria: Chemical features, role and enzymes and genes involved in their biosynthesis. The Physiology of Microalgae. 565-590.

[7] Delattre, C., Pierre, G., Laroche, C., Michaud, P., 2016. Production, extraction and characterization of microalgal and cyanobacterial exopolysaccharides. Biotechnology Advances. 34, 1159-1179.

[8] Tischer, R.G., Moore, B.G., 1964. An extracellular polysaccharide produced by Palmella mucosa. Kütz. Archiv für Mikrobiologie. 49, 158-166.

[9] Bae, S.Y., Yim, J.H., Lee, H.K., Pyo, S., 2006. Activation of murine peritoneal macrophages by sulfated exopolysaccharide from marine microalga Gyrodinium impudicum (strain KG03): Involvement of the NF- $\kappa \mathrm{B}$ and JNK pathway. International Immunopharmacology. 6, 473-484. [10] Chen, B., You, W., Huang, J., Yu, Y., Chen, W., 2010. Isolation and antioxidant property of the extracellular polysaccharide from Rhodella reticulate. World Journal of Microbiology and Biotechnology. 26, 833-840.

[11] Guzmán, S., Gato, A., Lamela, M., Freire-Garabal, M., Calleja, J.M., 2003. Antiinflammatory and immunomodulatory activities of polysaccharide from Chlorella stigmatophora and Phaeodactylum tricornutum. Phytotherapy Research. 17, 665-670.

[12] Majdoub, H., Mansour, M.B., Chaubet, F., Roudesli, M.S., Maaroufi, R.M., 2009. Anticoagulant activity of a sulfated polysaccharide from the green alga Arthrospira platensis. Biochimica et Biophysica Acta. 1790, 1377-1381.

[13] Najdenski, H.M., Gigova, L.G., Iliev, I.I., Pilarski, P.S., Lukavský, J., Tsvetkova, I.V., Ninova, M.S., Kussovski, V.K., 2013. Antibacterial and antifungal activities of selected microalgae and Cyanobacteria. International Journal of Food Science and Technology. 48, $1533-1540$. 
[14] Park, G.T., Go, R.E., Lee, H.M., Lee, G.A., Kim, C.W., Seo, J.W., Hong, W.K., Choi, K.C., Hwang, K.A., 2017. Potential anti-proliferative and immunomodulatory effects of marine microalgal exopolysaccharide on various human cancer cells and lymphocytes in vitro. Marine Biotechnology. 19, 136-146.

[15] Roussel, M., Villay, A., Delbac, F., Michaud, P., Laroche, C., Roriz, D., El Alaoui, H., Diogon, M., 2015. Antimicrosporidian activity of sulphated polysaccharides from algae and their potential to control honeybee nosemosis. Carbohydrate Polymers. 133, 213-220.

[16] De Philippis, R., Ena, A., Paperi, R., Sili, C., Vincenzini, M., 2000. Assessment of the potential of Nostoc strains from the Pasteur culture collection for the production of polysaccharides of applied interest. Journal of Applied Phycology. 12, 401-407.

[17] De Philippis, R., Margheri, M.C., Materassi, C., Vincenzini, M., 1998. Potential of Unicellular Cyanobacteria from Saline Environments as Exopolysaccharide Producers. Applied and Environmental Microbiology. 64, 1130-1132.

[18] Guillard, R.R.L., Ryther, J.H., 1962. Studies of marine planktonic diatoms: I. Cyclotella nana Hustedt, and Detonula Confervacea (cleve) Gran. Canadian Journal of Microbiology. 8, 229-239.

[19] Rippka, R., Coursin, T., Hess, W., Lichtle, C., Scanlan, D.J., Palinska, K.A., Iteman, I., Partensky, F., Houmard, J., Herdman, M. 2000. Prochlorococcus marinus Chisholm et al. 1992 subsp. pastoris subsp. nov. strain PCC 9511, the first axenic chlorophyll a2/b2-containing cyanobacterium (Oxyphotobacteria). International Journal of Systematic and Evolutionary Microbiology. 50, 1833-1847.

[20] Cawse, P.A., 1967. The determination of nitrate in soil solutions by ultraviolet spectrophotometry. Analyst. 92, 311-315. 
[21] A.P.H.A., 1971. Ultraviolet spectrophotometric methods. Standard methods for the examination of water and wastewater. American Public Health Association, Washington, DC. $237-239$.

[22] Patton, C.J., Crouch, S.R., 1977. Spectrophotometric and kinetics investigation of the Berthelot reaction for the determination of ammonia. Analytical Chemistry. 49, 464-469.

[23] Dubois, M., Gilles, K.A., Hamilton, J.K., Rebers, P.A., Smith, F., 1956. Colorimetric method for determination of sugars and related substances. Analytical Chemistry. 28, 350-356. [24] Pruvost, J., Cornet, J.F., 2012. Knowledge models for the engineering and optimization of photobioreactors. In: Microalgal Biotechnology: Potential and Production. Posten, C., Walter, C. (Eds.). 181-224.

[25] Villay, A., Laroche, C., Roriz, D., El Alaoui, H., Delbac, F., Michaud, P., 2013. Optimisation of culture parameters for exopolysaccharides production by the microalga Rhodella violacea. Bioresource Technology. 146, 732-735.

[26] Pierre, G., Graber, M., Rafiliposon, B.A., Dupuy, C., Orvain, F., De Crignis, M., Maugard, T., 2012. Biochemical composition and changes of extracellular polysaccharides (ECPS) produced during microphytobenthic biofilm development. Microbial Ecology. 63, 157-169.

[27] Pierre, G., Zhao, J.M., Orvain, F., Dupuy, C., Klein, G.L., Graber, M., Maugard, T., 2014. Seasonal dynamics of extracellular polymeric substances (EPS) in surface sediments of a diatom-dominated intertidal mudflat. Journal of Sea Research. 92, 26-35.

[28] Benjamini.Y., Hochberg. Y., 1995. Controlling the false discovery rate: a practical and powerful approach to multiple testing. Journal of the Royal Statistical Society. Series B. 57, 289-300.

[29] Guiry, M.D., Guiry, G.M., 2019. AlgaeBase. World-wide electronic publication, National University of Ireland, Galway. http://www.algaebase.org; searched on 04 March 2019. 
[30] Burki, F., 2014. The eukaryotic tree of life from a global phylogenomic perspective. Cold Spring Harb Perspect Biol. 6:a016147.

[31] Leliaert, F., Smith, D.R., Moreau, H., Herron, M.D., Verbruggen, H., Delwiche, C.F., De Clerck, O. (2012). Phylogeny and molecular evolution of the green algae. Critical Reviews in Plant Sciences. 31, 1-46.

[32] Worden, A.Z., Follows, M.J., Giovannoni, S.J., Wilken, S., Zimmerman, A.E., Keeling, P.J., 2015. Rethinking the marine carbon cycle: Factoring in the multifarious lifestyles of microbes. Science. 347, 6223, 1257594.

[33] Edgar, R.C., 2004. MUSCLE: multiple sequence alignment with high accuracy and high throughput. Nucleic Acids Research. 32, 1792-1797.

[34] Kumar, S., Stecher, G., and Tamura, K., 2016. MEGA7: Molecular Evolutionary Genetics Analysis Version 7.0 for Bigger Datasets. Molecular Biology and Evolution. 33, 1870-1874.

[35] Letunic, I., Bork, P., 2016. Interactive tree of life (iTOL) v3: an online tool for the display and annotation of phylogenetic and other trees. Nucleic Acids Research. 44, 242-245.

[36] Phlips, E.J., Zeman, C., Hansen, P., 1989. Growth, photosynthesis, nitrogen fixation and carbohydrate production by a unicellular cyanobacterium, Synechococcus sp. (Cyanophyta). Journal of Applied Phycology. 1, 137-145.

[37] De Philippis, R., Sili, C., Paperi, R., Vincenzini, M., 2001. Exopolysaccharide-producing cyanobacteria and their possible exploitation: A review. Journal of Applied Phycology. 13, 293299.

[38] Arad, S.M., Lerental, Y.B., Dubinsky, O., 1992. Effect of nitrate and sulfate starvation on polysaccharide formation in Rhodella reticulata. Bioresource Technology. 42, 141-148.

[39] De Philippis, R., Vincenzini, M., 1998. Exocellular polysaccharides from Cyanobacteria and their possible applications. FEMS Microbiology Reviews. 22, 151-175. 
[40] Ge, H., Xia, L., Zhou, X., Zhang, D., Hu, C., 2014. Effects of light intensity on components and topographical structures of extracellular polysaccharides from the cyanobacteria Nostoc sp. Journal of Microbiology. 52, 179-183.

[41] Ge, H., Zhang, J., Zhou, X., Xia, L., Hu, C., 2014. Effects of light intensity on components and topographical structures of extracellular polymeric substances from Microcoleus vaginatus (Cyanophyceae). Phycologia. 53, 167-173.

[42] Markou, G., Nerantzis, E., 2013. Microalgae for high-value compounds and biofuels production: A review with focus on cultivation under stress conditions. Biotechnology Advances. 31, 1532-1542.

[43] Mishra, A., Jha, B., 2009. Isolation and characterization of extracellular polymeric substances from micro-algae Dunaliella salina under salt stress. Bioresource Technology. 100, $3382-3386$

[44] You, T., Barnett, S.M., 2004. Effect of light quality on production of extracellular polysaccharides and growth rate of Porphyridium cruentum. Biochemical Engineering Journal. $19,251-258$

[45] Cahoon, L., 1999. The role of benthic microalgae in neritic ecosystems. Oceanography and Marine Biology: an Annual Review. 37, 47-86.

[46] Martin-Jézéquel, V., Hildebrand, M., Brzezinski, M.A., 2000. Silicon metabolism in Diatoms: Implications for growth. Journal of Phycology. 36, 821-840.

[47] de Jesus Raposo, M.F., De Morais, A.M.M.B., De Morais, R.M.S.C., 2014. Influence of sulphate on the composition and antibacterial and antiviral properties of the exopolysaccharide from Porphyridium cruentum. Life Sciences. 101, 56-63.

[48] Fábregas, F., García, D., Fernandez-Alonso, M., Rocha, A.I., Gómez-Puertas, P., Escribano, J.M., Otero, A., Coll, J.M., 1999. In vitro inhibition of the replication of 
haemorrhagic septicaemia virus (VHSV) and african swine fever virus (ASFV) by extracts from marine microalgae. Antiviral Research. 44, 67-73.

[49] Soanen, N., Da Silva, E., Gardarin, C., Michaud, P., Laroche, C., 2016. Improvement of exopolysaccharide production by Porphyridium marinum. Bioresource Technology. 213, 231238.

[50] Percival, E., Foyle, R.A.J., 1979. The extracellular polysaccharides of Porphyridium cruentum and Porphyridium aerugineum. Carbohydrate Research. 72, 165-176.

[51] Elboutachfaiti, R., Delattre, C., Petit, E., Michaud, P., 2011. Polyglucuronic acids: Structures, functions and degrading enzymes. Carbohydrate Polymers. 84, 1-13.

[52] Kiemle, S.N., Domozych, D.S., Gretz, M.R., 2007. The extracellular polymeric substances of desmids (Conjugatophyceae, Streptophyta): chemistry, structural analyses and implications in wetland biofilms. Phycologia. 46, 617-627.

[53] Paulsen, B.S., Vieira, A.A.H., 1994. Structure of the capsular and extracellular polysaccharides produced by the desmid Spondylosium panduriforme (Chlorophyta). Journal of Phycology. 30, 638-641.

[54] Domozych, C.R., Plante, K., Blais, P., Paliulis, L., Domozych, D.S., 1993. Mucilage Processing and Secretion in the Green Alga Closterium. I. Cytology and Biochemistry. Journal of Phycology. 29, 650-659.

[55] Vieira, A.A.H., Paulsen, B.S., 1994. Studies on the composition of extracellular polysaccharide produced by Hyalotheca dissiliens (Desmidiaceae). 49, 535-538.

[56] Barberousse, H., Ruiz, G., Gloaguen, V., Lombardo, R.J., Djediat, C., Mascarell, G., Castaing, J.C., 2006. Capsular polysaccharides secreted by building façade colonisers: characterisation and adsorption to surfaces. Biofouling. 22, 361-370.

[57] Khandeparker, R.D.S., Bhosle, N.B., 2001. Extracellular polymeric substances of the marine fouling diatom amphora rostrata Wm.Sm. Biofouling. 17, 117-127. 
[58] Wustman, B.A., Gretz, M.R., Hoagland, K.D., 1997. Extracellular matrix assembly in diatoms (Bacillariophyceae). A model of adhesives based on chemical characterization and localization of polysaccharides from the marine diatom Achnanthes longipes and other Diatoms. Plant Physiology. 113, 1059-1069.

[59] Bendif, E.M., Probert, I., Hervé, A., Billard, C., Goux, D., Lelong, C., Cadoret, J.P., Véron, B., 2011. Integrative taxonomy of the Pavlovophyceae (Haptophyta): a reassessment. Protist. $162,738-761$.

[60] Lopes, D.C., Maurer, J.B.B., Stevan-Hancke, F.R., Oliveira, P.L.A., Zawadzki-Baggio, S.F., 2012. Chemical analysis of exopolysaccharide fractions and lipid compounds of the microalga Heterosigma akashiwo grown in vitro. Botanica Marina. 55, 565-580.

[61] Yim, J.H., Son, E., Pyo, S., Lee, H.K., 2003. Optimal conditions for the production of sulfated polysaccharide by marine microalga Gyrodinium impudicum strain KG03. Marine Biotechnology. 7, 331-338.

[62] Parikh, A., Madamwar, D., 2006. Partial characterization of extracellular polysaccharides from cyanobacteria. Bioresource Technology. 97, 1822-1827.

[63] Di Pippo, F., Ellwood, N.T.W., Gismondi, A., Bruno, L., Rossi, F., Magni, P., De Philippis, R., 2013. Characterization of exopolysaccharides produced by seven biofilm-forming cyanobacterial strains for biotechnological applications. Journal of Applied Phycology. 25, 1697-1708.

[64] Richert, L., Golubic, S., Guédès, R.L., Ratiskol, J., Payri, C., Guezennec, J., 2005. Characterization of exopolysaccharides produced by cyanobacteria isolated from polynesian microbial mats. Current Microbiology. 51, 379-384.

[65] Leandro, S.M., Gil, M.C., Delgadillo, I., 2003. Partial characterization of exopolysaccharides exudated by planktonic diatoms maintained in batch cultures. Acta Oecologica. 24, S49-S55. 
[66] Paulsen, B.S., Aslaksen, T., Freire-Nordi, C.S., Vieira, A.A.H., 1998. Extracellular polysaccharides from Ankistrodesmus Densus (Chlorophyceae). Journal of Phycology. 34, 638641.

[67] Gouvêa, S.P., Vieira, A.A.H., Lombardi, A.T., 2005. Copper and cadmium complexation by high molecular weight materials of dominant microalgae and of water from a eutrophic reservoir. Chemosphere. 60, 1332-1339.

[68] Allard, B., Casadevall, E., 1990. Carbohydrate composition and characterization of sugars from the green microalga Botryococcus Braunii. Phytochemistry. 29, 1875-1878.

[69] Myklestad, S., Haug, A., Larsen, B., 1972. Production of carbohydrates by the marine diatom Chaetoceros affinis var. willei (Gran) Hustedt. II. Preliminary investigation of the extracellular polysaccharide. Journal of Experimental Marine Biology and Ecology. 9, 137-144. [70] Paulsen, B.S., Haug, A., Myklestad, S., 1975. Structural studies of the extracellular polysaccharide produced by the diatom Chaetoceros curvisetus cleve. Organic Chemistry and Biochemistry. 29, 337-340.

[71] Urbani, R., Sist, P., Pletikapic, G., Misic, T., Svetlicic, V., Zutic, V., 2012. Diatom polysaccharides: extracellular production, isolation and molecular characterization. The Complex World of Polysaccharides. 345-370.

[72] Allard, B., Tazi, A., 1993. Influence of growth status on composition of extracellular polysaccharides from two Chlamydomonas species. Phytochemistry. 32, 41-47.

[73] Flaibani, A., Olsen, Y., Painter, T.J., 1989. Polysaccharides in desert reclamation: Compositions of exocellular proteoglycan complexes produced by filamentous blue-green and unicellular green edaphic algae. Carbohydrate Research. 190, 235-248.

[74] Bafana, A., 2013. Characterization and optimization of production of exopolysaccharide from Chlamydomonas reinhardtii. Carbohydrate Polymers. 95, 746-752. 
[75] Barclay, W.R., Lewin, R.A., 1985. Microalgal polysaccharide production for the conditioning of agricultural soils. Plant and Soil. 88, 159-169.

[76] Kaplan, D., Christiaen, D., Arad, S.M., 1987. Chelating properties of extracellular polysaccharides from Chlorella spp. Applied and Environmental Microbiology. 53, 2953-2956. [77] Moore, B.G., Tischer, R.G., 1964. Extracellular polysaccharides of algae: effects on lifesupport systems. Science. 145, 586-587.

[78] Maksimova, I.V., Bratkovskaya, L.B., Plekhanov, S.E., 2004. Extracellular carbohydrates and polysaccharides of the alga Chlorella pyrenoidosa Chick S-39. Biology Bulletin of the Russian Academy of Sciences. 31, 175-181.

[79] Hasui, M., Matsuda, M., Okutani, K., Shigeta, S., 1995. In vitro antiviral activities of sulfated polysaccharides from a marine microalga (Cochlodinium polykrikoides) against human immunodeficiency virus and other enveloped viruses. International Journal of Biological Macromolecules. 17, 293-297.

[80] Percival, E., Anisur Rahman, M., Weigel, H., 1980. Chemistry of the polysaccharides of the diatom Coscinodiscus nobilis. Phytochemistry. 19, 809-811.

[81] Liu, B., Sun, Z., Ma, X., Yang, B., Jiang, Y., Wei, D., Chen, F., 2015. Mutation Breeding of Extracellular Polysaccharide-Producing Microalga Crypthecodinium cohnii by a Novel Mutagenesis with Atmospheric and Room Temperature Plasma. International Journal of Molecular Sciences. 16, 8201-8212.

[82] Staats, N., Winder, B.D., Stal, L.J., Mur, L.R., 1999. Isolation and characterization of extracellular polysaccharides from the epipelic diatoms Cylindrotheca closterium and Navicula salinarum. European Journal of Phycology. 34, 161-169.

[83] Magaletti, E., Urbani, R., Sist, P., Ferrari, C.R., Cicero, A.M., 2004. Abundance and chemical characterization of extracellular carbohydrates released by the marine diatom 
Cylindrotheca fusiformis under N- and P-limitation. European Journal of Phycology. 39, 133142.

[84] Hu, C., Liu, Y., Paulsen, B.S., Petersen, D., Klaveness, D., 2003. Extracellular carbohydrate polymers from five desert soil algae with different cohesion in the stabilization of fine sand grain. Carbohydrate Polymers. 54, 33-42.

[85] Gaignard, C., Macao, V., Gardarin, C., Rihouey, C., Picton, L., Michaud, P., Laroche, C., 2018. The red microalga Flintiella sanguinaria as a new exopolysaccharide producer. Journal of Applied Phycology. 30, 2803-2814.

[86] Park, J.K., Kim, Z.H., Lee, C.G., Synytsya, A., Jo, H.S., Kim, S.O., Park, J.W., Park, Y., 2011. Characterization and immunostimulating activity of a water-soluble polysaccharide isolated from Haematococcus lacustris. Biotechnology and Bioprocess Engineering. 16, 10901098.

[87] Oertel, A., Aichinger, N., Hochreiter, R., Thalhamer, J., Lütz-Meindl, U., 2004. Analysis of mucilage secretion and excretion in Micrasterias (Chlorophyta) by means of immunoelectron microscopy and digital time lapse video microscopy. Journal of Phycology. $40,711-720$

[88] Bhosle, N.B., Sawant, S.S., Garg, A., Wagh, A.B., 1995. Isolation and partial chemical analysis of exopolysaccharides from the marine fouling diatom Navicula subinflata. Botanica Marina. 38, 33-110.

[89] Domozych, D.S., Kort, S., Benton, S., Yu, T., 2005. The extracellular polymeric substance of the green alga Penium margaritaceum and its role in biofilm formation. Biofilms. 2, 129144.

[90] Geresh, S., Arad, S.M., Levy-Ontman, O., Zhang, W., Tekoah, Y., Glaser, R., 2009. Isolation and characterization of poly- and oligosaccharides from the red microalga Porphyridium sp. Carbohydrate Research. 344, 343-349. 
[91] Capek, P., Matulová, M., Combourieu, B., 2008. The extracellular proteoglycan produced by Rhodella grisea. International Journal of Biological Macromolecules. 43, 390-393.

[92] Cohen, A.K., Arad, S.A., 1998. Biosynthesis of the cell wall polysaccharide in the red microalga Rhodella Reticulata. Israel Journal of Plant Sciences. 46, 147-153

[93] Mohamed, Z.A., 2008. Polysaccharides as a protective response against microcystininduced oxidative stress in Chlorella vulgaris and Scenedesmus quadricauda and their possible significance in the aquatic ecosystem. Ecotoxicology. 17, 504.

[94] Freire-Nordi, C.S., Vieira, A.A.H., Nakaie, C.R., Nascimento, O.R., 2006. Effect of polysaccharide capsule of the microalgae Staurastrum iversenii var. americanum on diffusion of charged and uncharged molecules, using EPR technique. Brazilian Journal of Physics. 36, $75-82$.

[95] Giroldo, D., Vieira, A.A.H., Paulsen, B.S., 2003. Relative increase of deoxy sugars during microbial degradation of an extracellular polysaccharide released by a tropical freshwater Thalassiosira Sp. (Bacillariophyceae). Journal of Phycology. 39, 1109-1115.

[96] Lama, L., Nicolaus, B., Calandrelli, V., Manca, M.C., Romano, I., Gambacorta, A., 1996. Effect of growth conditions on endo- and exopolymer biosynthesis in Anabaena cylindrica 10 C. Phytochemistry. 42, 655-659.

[97] Moreno, J., Vargas, M.A., Olivares, H., Rivas, J., Guerrero, M.G., 1998. Exopolysaccharide production by the cyanobacterium Anabaena sp. ATCC 33047 in batch and continuous culture. Journal of Biotechnology. 60, 175-182.

[98] Nicolaus, B., Panico, A., Lama, L., Romano, I., Manca, M.C., De Giulio, A., Gambacorta, A., 1999. Chemical composition and production of exopolysaccharides from representative members of heterocystous and non-heterocystous cyanobacteria. Phytochemistry. 52, 639-647. [99] Sangar, V.K., Dugan, P.R., 1972. Polysaccharide produced by Anacystis nidulans: Its ecological implication. Applied Microbiology. 24, 732-734. 
[100] Sudo, H., Burgess, J.G., Takemasa, H., Nakamura, N., Matsunaga, T., 1995. Sulfated exopolysaccharide production by the halophilic cyanobacterium Aphanocapsa halophytia. Current Microbiology. 30, 219-222.

[101] Li, P., Liu, Z., Xu, R., 2001. Chemical characterisation of the released polysaccharide from the cyanobacterium Aphanothece halophytica GR02. Journal of Applied Phycology. 13, $71-77$.

[102] Nie, Z.Y., Xia, J.I., Levert, J.M., 2002. Fractionation and characterization of polysaccharides from cyanobacterium Spirulina (Arthrospira) maxima in nitrogen-limited batch culture. Journal of Central South University of Technology. 9, 81-86.

[103] Trabelsi, L., M'sakni, N.H., Ben Ouada, H., Bacha, H., Roudesli, S., 2009. Partial characterization of extracellular polysaccharides produced by cyanobacterium Arthrospira platensis. Biotechnology and Bioprocess Engineering. 14, 27-31.

[104] Fischer, D., Schlösser, U.G., Pohl, P., 1997. Exopolysaccharide production by cyanobacteria grown in closed photobioreactors and immobilized using white cotton toweling. Journal of Applied Phycology. 9, 205-213.

[105] Vincenzini, M., De Philippis, R., Sili, C., Materassi, R., 1993. Stability of molecular and rheological properties of the exopolysaccharide produced by Cyanospira capsulata cultivated under different growth conditions. Journal of Applied Phycology. 5, 539-541.

[106] De Philippis, R., Margheri, M.C., Pelosi, E., Ventura, S., 1993. Exopolysaccharide production by a unicellular cyanobacterium isolated from a hypersaline habitat. Journal of Applied Phycology. 5, 387-394.

[107] Rossi, F., Micheletti, E., Bruno, L., Adhikary, S.P., Albertano, P., De Philippis, R., 2012. Characteristics and role of the exocellular polysaccharides produced by five Cyanobacteria isolated from phototrophic biofilms growing on stone monuments. Biofouling. 28, 215-224. 
[108] Tease, B., Jürgens, U.J., Golecki, J.R., Heinrich, U.R., Rippka, R., Weckesser, J., 1991. Fine-structural and chemical analyses on inner and outer sheath of the cyanobacterium Gloeothece sp. PCC 6909. Antonie van Leeuwenhoek. 59, 27-34.

[109] Gloaguen, V., Morvan, H., Hoffmann, L., 1995. Released and capsular polysaccharides of Oscillatoriaceae (Cyanophyceae, Cyanobacteria). Algological Studies. 78, 53-69.

[110] Forni, C., Telo, F.R., Caiola, M.G., 1997. Comparative analysis of the polysaccharides produced by different species of Microcystis (Chroococcales, Cyanophyta). Phycologia. 36, 181-185.

[111] Huang, Z., Liu, Y., Paulsen, B.S., Klaveness, D., 1998. Studies on polysaccharides from three edible species of Nostoc (Cyanobacteria) with different colony morphologies: Comparison of monosaccharide compositions and viscosities of polysaccharides from field colonies and suspension cultures. Journal of Phycology. 34, 962-968.

[112] Volk, R.B., Venzke, K., Blaschek, W., 2006. Structural investigation of a polysaccharide released by the cyanobacterium Nostoc insulare. Journal of Applied Phycology. 19, 255-262. [113] Panoff, J.M., Priem, B., Morvan, H., Joset, F., 1988. Sulphated exopolysaccharides produced by two unicellular strains of Cyanobacteria, Synechocystis PCC 6803 and 6714 . Archives of Microbiology. 150, 558-563. 